\title{
Molecular Simulations of Surfactant Adsorption on Iron Oxide from Hydrocarbon Solvents
}

\author{
Pablo Navarro Acero, ${ }^{\dagger, \ddagger}$ Stephan Mohr,,,,$+ \ddagger$ Marco Bernabei, $"$ Carlos

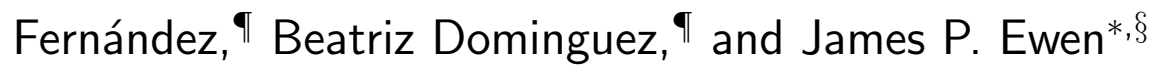 \\ $†$ †extmol (Bytelab Solutions SL), Carrer de Roc Boronat 117, 08018 Barcelona, Spain \\ $\ddagger$ Barcelona Supercomputing Center (BSC-CNS), Carrer Jordi Girona 29, 08034 Barcelona, \\ Spain \\ ФRepsol Technology Lab, DC Technology 8 Corporate Venturing, Agustín de Betancourt \\ s/n, 28935 Mostoles, Madrid, Spain \\ $\S$ Department of Mechanical Engineering, Imperial College London, South Kensington \\ Campus, London, SWY 2AZ, U.K. \\ E-mail: stephan.mohr@nextmol.com; j.ewen@imperial.ac.uk
}

\begin{abstract}
The performance of lubricant additives, such as organic friction modifiers (OFMs), depends critically on their ability to adsorb onto the surfaces of moving components and form protective self-assembled layers (SAMs). Therefore, understanding the relationship between the concentration of the additive in the base oil and the resulting surface coverage is extremely important for lubricant formulations, as well as many other surfactant applications. Here, we use molecular dynamics (MD) simulations to study the adsorption isotherms of three different OFMs, stearic acid (SA), glycerol monoostearate (GMS), and glycerol monooleate (GMO), onto a hematite surface from hydrocarbon solvents, $n$-hexadecane and poly- $\alpha$-olefin (PAO). First, we calculate the
\end{abstract}


potential of mean force (PMF) of the adsorption process using MD simulations with the adaptive biasing force $(\mathrm{ABF})$ algorithm. Our $\mathrm{MD}$ simulations show that $\mathrm{SA}$ has the weakest adsorption energy on hematite, followed by GMS, and finally GMO, due to the increasing number of functional groups available to bind to the surface. We also estimate the area occupied by each OFM molecule on the surface in the high-coverage limit using MD simulations of the annealing of OFM films with different initial surface coverages. We obtain a similar hard-disk area for GMS and GMO, but a lower value for SA, which is due to its smaller headgroup size. Based on the adsorption energy and surface area, we determine the corresponding adsorption isotherms using the molecular thermodynamic theory (MTT), which agree well with one available experiments experimental data-set for SA. Two other experimental data-sets for SA require lateral interactions between surfactant molecules to be accounted for. SA forms monolayers with lower surface coverage than GMO and GMS at low concentrations (due to a smaller adsorption energy), but also has the highest plateau coverage (due to a smaller hard-disk area). We validate the adsorption energies from the MD simulations using high frequency reciprocating rig (HFRR) friction experiments with different concentrations of the OFMs in PAO. We use the Jahanmir and Beltzer model to estimate the surface coverage at each concentration and the adsorption energy of each OFM from the HFRR friction data. For OFMs with saturated tailgroups (SA and GMS), we obtain good agreement between the predictions made by the simulations and the experiments. The MD simulation and experimental results deviate for OFMs containing $Z$-unsaturated tailgroups (GMO), with the former suggesting stronger adsorption for GMO than GMS, while the latter predicts the opposite trend. We suggest that this is can be attributed to the higher steric barrier of adsorption of the OFMs with kinked $Z$-unsaturated tailgroup through a partially formed monolayer, an aspect which was not captured in the current simulations. This study demonstrates that MD simulations with the ABF algorithm, alongside MTT, are an accurate and efficient tool to predict adsorption isotherms at solid-liquid interfaces. 


\section{Introduction}

The adsorption and self-assembly of surfactants at solid-liquid interfaces plays a key role in a wide range of technological processes, from nanoparticle stabilisation to ore flotation. ${ }^{1}$ In tribology, the adsorption of polar lubricant additives from nonpolar base oils onto solid surfaces is critical to their effective operation. ${ }^{2}$ For example, organic friction modifiers (OFMs), such as stearic acid (SA), adsorb and form thin ( $\sim 2 \mathrm{~nm})$ self-assembled monolayers (SAMs) on steel surfaces, which significantly reduce friction and wear. ${ }^{3}$ Similarly, inorganic friction modifiers, such as molybdenum dialkyldithiocarbamate (MoDTC), adsorb and decompose to form thin $(\sim 1 \mathrm{~nm})$, low-friction lamellar molybdenum disulphide films on steel. ${ }^{4}$ Moreover, antiwear additives, such as zinc dialkyldithiophosphate (ZDDP), adsorb, form SAMs, ${ }^{5}$ and decompose on steel surfaces to form thick $(\sim 100 \mathrm{~nm})$ protective tribofilms, composed mostly of metal phosphates. ${ }^{6}$

Modern lubricant formulations contain at least ten different types of additive dissolved in a blend of base oils. ${ }^{7}$ Combinations of different additives often behave antagonistically, that is the additive blend gives poorer performance than any of the individual additives alone at equal concentration. ${ }^{8}$ Prominent examples of antagonistic lubricant additive combinations include overbased calcium sulphonate detergents with ZDDP,${ }^{9}$ succinimide dispersants with ZDDP ${ }^{10}$ and MoDTC with ester OFMs. ${ }^{11}$ Many of these antagonistic interactions are due to competitive adsorption processes, whereby the most surface-active component occupies the majority of surface sites and inhibits the action of the other additives. ${ }^{8}$ Therefore, it is important to understand the relative adsorption strength of the various lubricant additives from base oils on steel surfaces.

OFMs are one of the earliest and most widely studied types of lubricant additive. ${ }^{12}$ Hardy and Doubleday ${ }^{13}$ were the first to suggest that OFM friction reduction was due to the formation of vertically-oriented surfactant monolayers on the sliding surfaces. They found that 
fatty acids with longer chain length $\left(\mathrm{C}_{4}-\mathrm{C}_{12}\right)$ showed lower boundary friction and that fatty acids gave lower friction than fatty alcohols ${ }^{13}$. Bowden and Leben ${ }^{14}$ showed that the friction of $\mathrm{SA}\left(\mathrm{C}_{18}\right)$ monolayers deposited on steel surfaces with the Langmuir-Blodgett technique was initially the same as that for SAMs formed from a hydrocarbon base oil. Fowkes ${ }^{15}$ showed that OFM adsorption strength from hydrocarbon solution onto steel varied in the order carboxylic acid $>$ amine $>$ alcohol, and Groszek ${ }^{16}$ showed that this correlated with antiwear performance. Rowe ${ }^{17}$ also found that adsorption strength and antiwear performance were correlated for OFMs, but with a slightly different order (amine > carboxylic acid > alcohol). Using this data, Rowe developed a mathematical model to calculate thermodynamic quantities describing adsorption from experimentally-obtained wear rates. ${ }^{17}$ Spikes and Cameron ${ }^{18}$ showed that adsorption and lubricant failure both had a similar dependence on fatty amine concentration and temperature, suggesting that lubricant failure could be due to desorption of the OFM molecules. Jahanmir and Beltzer ${ }^{19}$ studied the adsorption and friction behaviour of a wide range of carboxylic acid, ester, alcohol, amine, and amide OFMs with different alkyl tailgroup lengths, branching, and unsaturation. They found that additives that adsorbed more strongly generally gave lower friction. ${ }^{19}$ Jahanmir and Beltzer ${ }^{20}$ also built upon the model due to Rowe ${ }^{17}$ to describe the relationship between the friction coefficient and adsorption energy for a two-component lubricant (i.e. base oil and one additive), assuming that the minimum friction coefficient observed at high OFM concentration was due to the formation of a close-packed SAM. ${ }^{20}$

Recent depletion isotherm and polarised neutron reflectometry (PNR) experiments by Wood et al. ${ }^{21}$ for the adsorption from $n$-dodecane onto iron oxide surfaces have shown that OFMs with linear, saturated tailgroups (SA) do form monolayers with a high surface coverage, similar to that expected for a close-packed monolayer, at high concentration. Conversely, OFMs with kinked, $Z$-unsaturated tailgroups, such as oleic acid (OA), form monolayers with much lower surface coverage. This observation explained the higher friction coefficient for 
OA than SA reported in nanoscale atomic force microscopy (AFM) by Lundgren et al. ${ }^{22}$ and microscale surface forces apparatus (SFA) experiments by Ruths et al. ${ }^{23}$ The same observations were made at the macroscale by Campen et al. ${ }^{24}$ for OA and glycerol mono-oleate (GMO) compared to SA and glycerol mono-stearate (GMS) using steel substrates and an $n$-hexadecane solvent. Using a range of OFMs with different headgroups and tailgroups, Fry et al. ${ }^{25}$ showed through a combination of quartz crystal microbalance (QCM), ellipsometry, and tribology experiments that OFMs which formed higher coverage monolayers gave lower initial friction coefficients.

In recent years, molecular simulations have provided additional insights into the nanoscale behaviour of a wide range of lubricant additives, including OFMs. ${ }^{26,27}$ The adsorption energy of lubricant additives on metal oxide surfaces can be accurately determined with first principles methods such as density functional theory (DFT). ${ }^{28-30}$ However, due to its very high computational cost, DFT is typically performed in vacuum at zero temperature, and can only capture the enthalpy contributions to the adsorption free energy. For many surfactants at solid-liquid interfaces, entropy plays a central role in the adsorption process. ${ }^{31}$ These entropic contributions can be calculated using classical molecular dynamics (MD) simulations, ${ }^{32}$ which can be used to access much larger time and length scales than DFT. Nevertheless, capturing the adsorption and self-assembly processes from solution using realistic surfactant concentrations represents a considerable computational challenge. ${ }^{27}$

Until recently, a certain surface coverage of surfactant molecules molecules on the solid surfaces was usually assumed in MD simulations of OFMs. ${ }^{26,27}$ However, Chia et al. ${ }^{33}$ and Jaishankar et al. ${ }^{34}$ used MD simulations to study the adsorption of surfactants on iron oxide surfaces from hydrocarbon solvents. Chia et al. ${ }^{33}$ studied the competitive adsorption of the fuel additive 2-[(3-(dimethylamino)propyl)amino]methyl-4-dodecyl-6-methyl-phenol and ethanol from $i$-octane onto the $\alpha-\mathrm{Fe}_{2} \mathrm{O}_{3}(0001)$ surface at different temperatures. They 
showed that, while the free energy of adsorption of the additive was approximately two times larger than that for a single ethanol molecule, a single additive needs to displace up to five ethanol molecules to strongly adsorb onto the surface. Jaishankar et al. ${ }^{34}$ studied SA adsorption from $n$-heptane and $n$-hexadecane onto $\mathrm{Fe}_{3} \mathrm{O}_{4}(111), \mathrm{Fe}_{3} \mathrm{O}_{4}(001)$, and hydroxylated $\mathrm{Fe}_{3} \mathrm{O}_{4}(001)$ surfaces at room temperature. They found that adsorption was stronger on the hydroxylated surface and adsorption from $n$-hexadecane was much slower than from $n$-heptane due to stronger alignment of the molecules on the surface. They used a combined approach using MD and molecular thermodynamic theory (MTT) to calculate adsorption isotherms, which agreed well with experimental data obtained with QCM. ${ }^{34}$ The theoretical MTT model for the adsorption of non-ionic surfactants, originally developed by Nikas et al., ${ }^{35}$ was applied in combination with MD for the first time by Sresht et al. ${ }^{36}$ to study surface tensions at a water-air interface. Since then, it has been used to study adsorption in many systems, such as sodium dodecyl sulphate (SDS) on graphene from water, ${ }^{37}$ SDS on $\alpha-\mathrm{Al}_{2} \mathrm{O}_{3}$ nanoparticles from water, ${ }^{38}$ and organomolybdenum friction modifiers on borondoped diamond-like-carbon (DLC) from $n$-heptane. ${ }^{39}$

Chia et al. ${ }^{33}$ and Jaishankar et al. ${ }^{34}$ extracted the potential of mean force (PMF) along the reaction coordinate using umbrella sampling (US) and the weighted histogram analysis method (WHAM). ${ }^{40,41}$ Even using modern simulation software and high performance computing (HPC) architectures, this method requires hundreds of nanoseconds of simulation time to obtain converged PMFs. For example, Jaishankar et al. ${ }^{34}$ used an overall simulation time of more than 1 us to obtain the PMF describing the adsorption of SA onto hematite from $n$-hexadecane. A potentially more efficient method to obtain the PMF for surfactants at solid-liquid interfaces is the adaptive biasing force (ABF) algorithm. ${ }^{42,43}$ The ABF method has been used to study adsorption at solid-liquid interfaces for a range of surfactants from water, such as SDS on graphene, ${ }^{37}$ chlorogenic acid on polydimethylsiloxane, ${ }^{44}$ lipoic acid on silver, ${ }^{45}$ peptides on gold, ${ }^{46}$ proteins on hydroxyapatite ${ }^{47}$ and graphene, ${ }^{48}$ ethylene glycols 
on calcite ${ }^{49}$ and hematite, ${ }^{50}$ and a wide range of organic molecules on carbon nanotubes, ${ }^{51}$ graphene, ${ }^{52}$ and graphene oxide. ${ }^{53}$

In this study, we compare the adsorption of different OFMs from hydrocarbon solvents onto iron oxide surfaces. More specifically, ABF-MD is used to study the adsorption of SA, GMS and GMO from $n$-hexadecane and hydrogenated 1-decene trimer onto the $\alpha$ - $\mathrm{Fe}_{2} \mathrm{O}_{3}(010)$ surface. We show that the OFM headgroup and tailgroup structure, as well as the solvent, significantly affect the adsorption behaviour. Once the adsorption PMF is obtained, the hard-disk area occupied by the molecules on the surface due to steric effects is estimated using separate annealing MD simulations. Subsequently, the relation between the bulk concentration of the OFMs in the solvent and the surface coverage, i.e. the adsorption isotherm, is determined using the MTT model. ${ }^{34,35,54}$ Finally, the Jahanmir and Beltzer model, ${ }^{20}$ together with High Frequency Reciprocating Rig (HFRR) friction measurements, are used to experimentally validate the calculated adsorption energies. ${ }^{20,34}$

\section{Methods}

\section{System Setup}

The studied OFMs are SA, a carboxylic acid containing a saturated $\mathrm{C}_{18}$ tailgroup, GMS, a glyceride mono-ester containing a saturated $\mathrm{C}_{18}$ tailgroup, and GMO, a glyceride ester containing a $\mathrm{C}_{18}$ tailgroup with a $Z$-double bond in the middle of the chain. While $\mathrm{SA}$ is commonly used as a prototypical OFM, it is no longer used in commercial formulations due to its acidic nature, and has been replaced with amines, amides, and esters, such as GMS and GMO. ${ }^{12}$ The molecular structures of the OFMs are shown in Fig. 1a.

There has been some debate in the literature as to whether GMO and GMS adsorb 


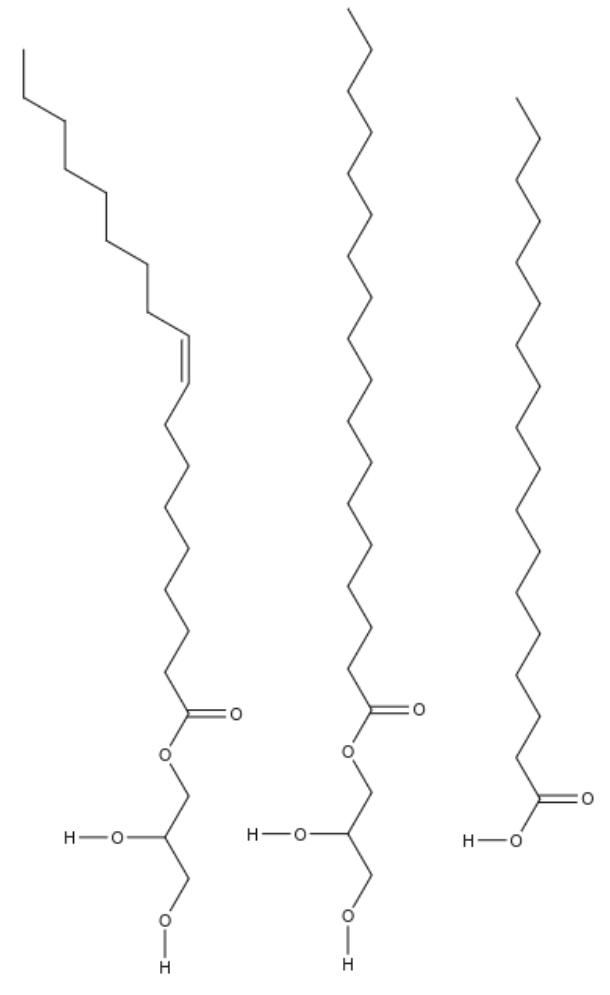

(a)

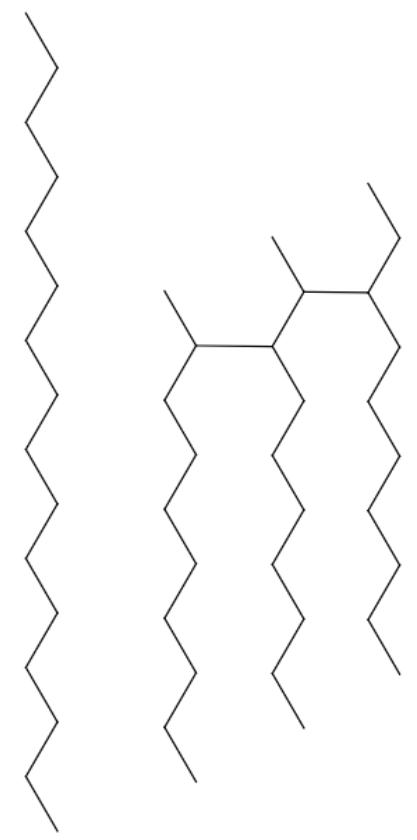

(b)

Figure 1: a) OFM molecular structures, from left to right: GMO, GMS and SA. b) Solvent molecular structures, from left to right: $n$-hexadecane and hydrogenated 1-decene trimer (PAO4). 
and reduce friction as intact glyceride esters or are hydrolysed to form the corresponding carboxylic acid that adsorbs, self-assembles, and reduces friction. ${ }^{12,55-57}$ Recent experiments have shown that the initial friction of GMO solutions is much lower than OA on silica surfaces. ${ }^{25}$ Therefore, in this study, we consider the adsorption of intact GMO and GMS molecules.

For the hydrocarbon solvent, $n$-hexadecane was compared to hydrogenated 1-decene trimer. While $n$-hexadecane is commonly used as a model base oil in adsorption and boundary friction experiments, ${ }^{24,25}$ hydrogenated 1-decene trimer is the primary constituent of commercial PAO4 base oil, a poly- $\alpha$-olefin with a kinematic viscosity of $4 \mathrm{cSt}$ at $100{ }^{\circ} \mathrm{C} .{ }^{58}$ The molecular structures of the base oils are shown in Fig. $1 \mathrm{~b}$.

All of the systems for the PMF adsorption calculation were constructed using the Materials and Processes Simulations (MAPS) platform from Scienomics SARL, whereas the systems to estimate the hard-disk area on the surface were constructed using proprietary scripting.

When exposed to air, steel forms passivating iron oxide surfaces, such as hematite $(\alpha-$ $\mathrm{Fe}_{2} \mathrm{O}_{3}$ ). ${ }^{59}$ Two slabs of $\alpha-\mathrm{Fe}_{2} \mathrm{O}_{3}$ with dimensions (xyz) of approximately $55 \AA \times 55 \AA \times 12$ $\AA$ were used as the substrates, with the (010) plane exposed to the liquid phase. Periodic boundary conditions were imposed in the $x$ - and $y$-directions. Initially, the two hematite slabs were separated by $120 \AA$ of vacuum space. For the adsorption calculations, a single OFM molecule was placed at the centre of the simulation cell, and either $700 n$-hexadecane or $373 \mathrm{PAO}$ molecules were randomly distributed between the slabs using the amorphous builder plugin in MAPS, see Figure 2. This gave a similar total number of $\mathrm{C}$ atoms ( 11200) for both systems. Two systems were used for the hard-disk area calculations (see Supporting Information). In the first set-up (dimerisation simulations), two OFM molecules were placed close to the surface of one of the hematite slabs, using the same system size and number of solvent molecules as in the adsorption calculations. In the second set-up (annealing simulations), one hematite slab was covered a with pre-formed monolayer of OFM 
molecules with different surface coverages. The number of OFM molecules included varied from 106 to 198 and the system size was approximately $55 \AA \times 55 \AA \times 100 \AA$. This yields initial surface coverages, $\Gamma=3.5-6.5 \mathrm{~nm}^{-2}$

The base oil and OFM molecules were modeled using the all-atom long chain-optimised potential for liquid simulations (L-OPLS-AA) ${ }^{61}$ force field. This is an updated version of OPLS-AA ${ }^{62}$ in which some of the parameters for $\mathrm{C}$ and $\mathrm{H}$ atoms are adjusted to accurately reproduce the experimental density and viscosity of long chain alkanes. ${ }^{63}$ Parameters for the other atoms remain unchanged from the orignal OPLS-AA forc field. ${ }^{62}$ The Lennard-Jones parameters and partial charges for the $\mathrm{Fe}$ and $\mathrm{O}$ atoms in the $\alpha-\mathrm{Fe}_{2} \mathrm{O}_{3}$ surface were taken from Berro et al. ${ }^{64}$ Moreover, harmonic bonds with a force constant of $130 \mathrm{kcal} \mathrm{mol}^{-1} \AA^{-1}$ were added between atoms within $0.3 \mathrm{~nm}$ of each other in their lattice positions. ${ }^{64}$ Bond lengths were taken from X-ray diffraction experiments by Blake et al. ${ }^{65}$ This combination of force fields has already been successfully used by Ewen et al ${ }^{66}$ in nonequilibrium molecular dynamics (NEMD) simulations of OFM friction.

It is noteworthy that recent DFT calculations have shown that strong molecule-surface interactions can occur for OFMs on iron oxide surfaces in the absence of solvent molecules. ${ }^{29}$ DFT calculations have also been used to parametrize classical force fields to more accurately represent amide-surface chemisorption. ${ }^{67}$ However, this task has not yet been executed for the OFM molecules of interest in this study.

\section{Simulation procedure}

MD simulations were performed using the Large-scale Atomic/Molecular Massively Parallel Simulator (LAMMPS) software. ${ }^{68}$ The velocity-Verlet algorithm ${ }^{69}$ was used to integrate the MD equations of motion. A time step of $1 \mathrm{fs}$ was used for all of the simulations and all bonds involving $\mathrm{H}$ atoms were fixed using the SHAKE algorithm. ${ }^{70}$ Long-range electrostatic interactions were calculated using the slab implementation of the particle-particle, particle- 


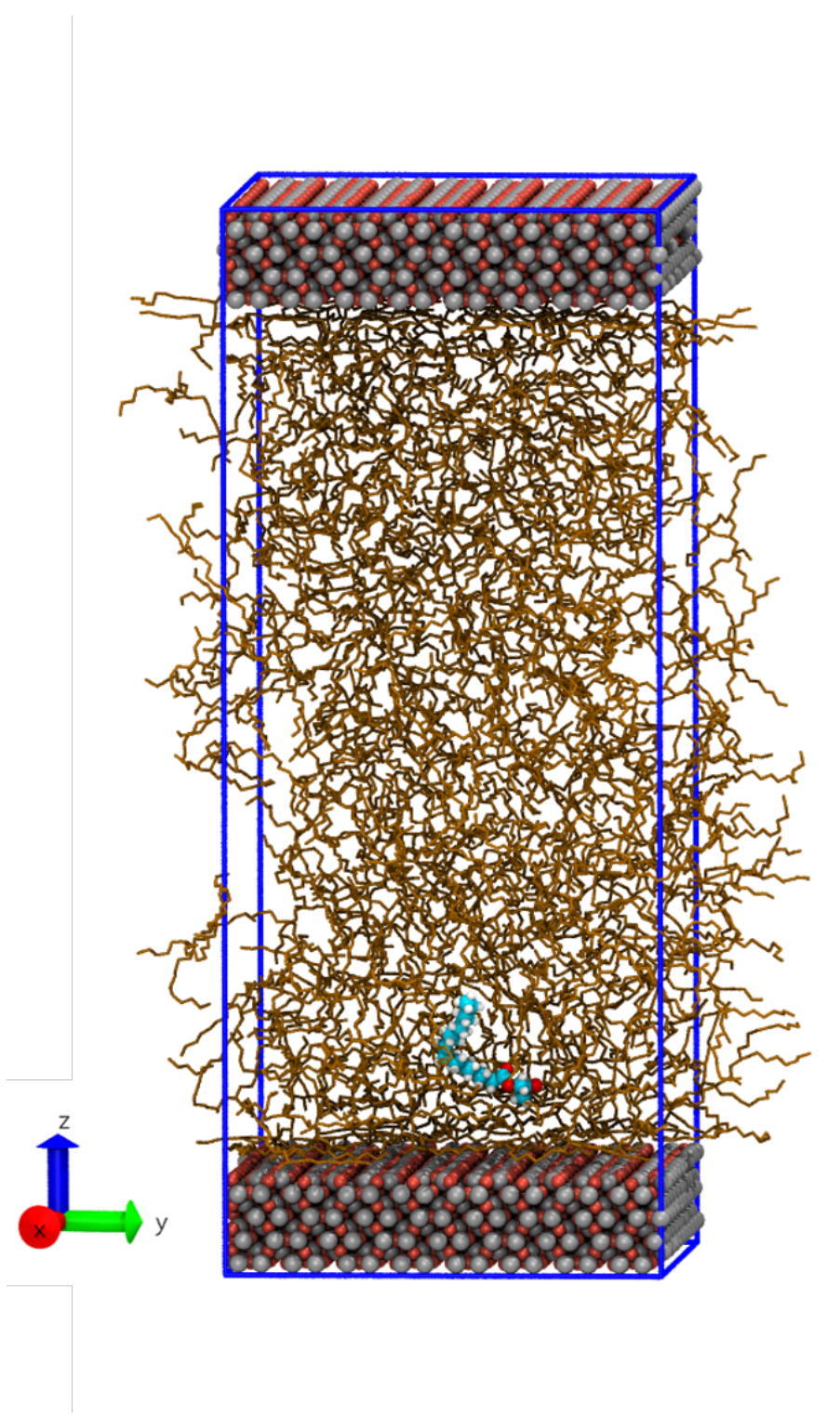

Figure 2: VMD visualization ${ }^{60}$ of a representative system used for the adsorption simulations (GMO in $n$-hexadecane). OFM atoms shown as beads: $\mathrm{O}$ atoms in red, $\mathrm{H}$ atoms in white, $\mathrm{C}$ atoms from OFM in blue and Fe atoms in gray. Solvent molecules shown as lines, $\mathrm{C}$ atoms shown in brown, $\mathrm{H}$ atoms not shown. The dark blue box depicts the simulation cell, exhibiting periodic boundary conditions in the $x$ and $y$ directions (i.e. parallel to the surfaces). 
mesh (PPPM) algorithm with a relative force accuracy of $10^{-4} .{ }^{71}$ The PMFs were extracted using the $\mathrm{ABF}$ method ${ }^{42,43}$ with the collective variables (colvars) module implemented in LAMMPS. ${ }^{72}$

Before starting the PMF production runs, the systems were energy minimized using the conjugate gradient algorithm, with all of the hematite atoms being fixed in place. Subsequently, the system was equilibrated at $300 \mathrm{~K}$ and $101 \mathrm{kPa}$ for $2 \mathrm{~ns}$. The outer layer of atoms in the bottom wall of hematite were fixed in all directions, whereas the outer layer of the top wall was fixed only in the $x$ - and $y$-directions, but allowed to move in the $z$-direction during the equilibration phase. These outer layers consisted of all of the $\mathrm{Fe}$ and $\mathrm{O}$ atoms that were $1 \AA$ or less from the bottom and the top of the simulation box respectively (440 atoms in both cases).

The pressure was controlled during the equilibration phase by applying a constant force to the outer layer of atoms in the top wall. After equilibration, the outer layers of both hematite slabs were fixed in place for the subsequent PMF production simulations. At the start of the equilibration phase, the velocities of the solvent, OFM and non-fixed hematite atoms were initialized using with a Gaussian distribution of velocities corresponding to a temperature of 300 K. The temperature was then controlled using the Nosè-Hoover ${ }^{73,74}$ thermostat with a time relaxation constant of $0.1 \mathrm{ps}$. The thermostat was applied to all of the non-fixed atoms.

The PMF was calculated using the ABF method, an importance sampling algorithm developed by Pohorille and Darve. ${ }^{42,75}$ The algorithm is based on the computation of the mean force along a certain collective variable (colvar), which is divided into small bins. The mean force is then cancelled out by an equal and opposite biasing force. This allows the system to move freely along that colvar, overcoming energy barriers and recovering ergodicity. The mean force profiles converge once the relevant range of the colvar has been explored and sufficient samples have been accumulated within each bin. The convergence of this method is subservient to the efficient exploration of the phase space orthogonal to the chosen colvar. In our case, this corresponds to the different orientations and conformations of the OFM 
molecules for each distance to the surface. Finally, the PMF is calculated from the mean force profile, since the average force exerted by the system onto the colvar is the gradient of the free energy profile along the colvar with a sign change:

$$
\frac{d A}{d \xi}\left(\xi_{0}\right)=-\left\langle F\left(\xi_{0}\right)\right\rangle,
$$

where $A$ is the free energy profile as a function of the colvar $\xi$ and $F$ is the force exerted by the system onto the colvar. ${ }^{42,75}$ In principle, $\xi$ is a continuous variable and equation 1 applies for every value $\xi_{0}$. In practice, the colvar is divided into small bins of width $\Delta \xi$ and samples are aquired for each bin during the simulation to compute the mean force in the interval $\left(\xi_{0}, \xi_{0}+\Delta \xi\right)$. Then, the mean value of those samples for each bin is used to compute the gradient and, through integration, the PMF. The review by review by Comer et al. ${ }^{76}$ provides a more detailed description of the $\mathrm{ABF}$ method, together with recommendations and good practices.

\section{Adsorption PMF}

To estimate the PMF that captures the adsorption process, the colvar was defined as the projection onto the $z$-axis of the distance between the center of mass (COM) of the OFM polar headgroup and the COM of the topmost layer of atoms of the bottom hematite surface. To accelerate convergence, the relevant range of the collective variable can be divided into several regions or "windows" to which the ABF algorithm is applied simultaneously. This approach is known as the stratification scheme. ${ }^{77}$ The length of these windows should not be too small, as restricting the range of the colvar too much in a single simulation may lead to a slowdown in convergence or even a loss of ergodicity. ${ }^{76}$ In other words, the simulation should visit the same configurations and in the same proportion as it would do using a much larger window, so that the artificial limit to the colvar explored in each window does not introduce a bias in the sampling. 
In this case, the window closest to the surface should be large enough so as to avoid that the tail remains intermingled in the first maximum of the solvent layering while the colvar is being explored. Otherwise all the samples acquired for the first window would correspond to the same tail position, biasing the exploration of the phase space. Due to these considerations, the calculations for SA and GMS were run with three windows: from 1.8-2.3 to $20 \AA, 20$ to $30 \AA$ and 30 to $40 \AA$, since their saturated linear tailgroups are more extended than the kinked $Z$-unsaturated tailgroups in GMO. Therefore, four windows were used for GMO: 2.3 to $10 \AA, 10$ to $20 \AA, 20$ to $30 \AA$ and 30 to $40 \AA$. The initial configurations for each window were obtained using steered molecular dynamics (SMD), ${ }^{78}$ as implemented in the LAMMPS colvars package. ${ }^{72}$ The force constant of the applied harmonic biasing potential was $10 \mathrm{kcal} \mathrm{mol}{ }^{-1} \AA^{-2}$, and the equilibrium distance was moved at a velocity of $4 \mathrm{~m} \mathrm{~s}^{-1}$ from the initial position of the molecule towards the surface. One initial condition was saved for each window and was equilibrated for 2 ns before applying the $\mathrm{ABF}$ algorithm to each window. The chosen width of the colvar bins was $\Delta \xi=0.05 \AA$.

The ABF algorithm was applied to each window until convergence was reached. As for other importance sampling techniques, the assessment of convergence is not trivial. ${ }^{76}$ Several necessary conditions for convergence were monitored to determine a reasonable trade-off between computational cost and precision. These included the uniform increase of the samples across bins, the absence of further changes to the PMF, and the continuity of the mean force between different windows (see the Supporting Information). The mean force converged more slowly in the windows that were close to the surface due to the existence of several orientations and conformations that correspond to different values of the mean force. The total simulation times required to obtain converged PMFs varied between 165 ns and 750 ns for windows adjacent to the surface and between $75 \mathrm{~ns}$ and $150 \mathrm{~ns}$ for the others. The calculations using $n$-hexadecane converged more quickly than those with PAO4. This is consistent with the observations of Jaishankar et al., ${ }^{34}$ who found that calculations converged 
more quickly for $n$-heptane than $n$-hexadecane, due to shorter relaxation times of the former.

\section{Hard-disk area}

To obtain a simulated adsorption isotherm using MTT, it is also necessary to estimate the area that each adsorbed molecule occupies on the surface. As explained by Nikas et al., ${ }^{35}$ several difficulties arise concerning the estimation of the hard-disk area of each molecule on the surface due to steric effects. For instance, this area depends on the molecular conformations accessible to the adsorbed surfactant, which will depend on the surface coverage. As a result, the effective cross-sectional area will be coverage-dependent. In practice, it is usually sufficient to estimate the hard-disk area at high concentration and thus high coverage, since this is where the steric repulsion of adsorbed molecules limits further increases to the surface coverage.

OFMs are known to form vertically-orientated SAMs on iron oxide surfaces. ${ }^{12,13}$ Therefore, the surfactant headgroup size will mostly determine the hard-disk area of the adsorbed molecule. Two approaches were used to estimate the hard-disk area occupied by the OFMs on the hematite surface. In the first approach, the hard-disk radius was estimated using the ABF method to calculate the PMF for the lateral interactions between OFMs. Two OFMs were adsorbed on the hematite surface and the projection onto the $x-y$ plane of the distance between the headgroups was used as colvar. Subsequently, the cross-sectional area occupied by each OFM on the hematite surface was estimated using the position of the PMF minimum, following the approach used by Jaishankar et al. ${ }^{79}$ for $\mathrm{SA}$ at the $n$-heptane-iron oxide interface and Sresht et al. ${ }^{36}$ for polyethylene glycol at the air-water interface. The results obtained using this first method can be found in the Supporting Information. In the second approach, annealing simulations of preformed OFM monolayers were performed. A similar approach to that described by Zhang et al. ${ }^{80}$ was used here. Different numbers of OFM molecules were placed close to the surface to form monolayers with a range of initial 
surface coverages. A subsequent equilibration of 2 ns allowed the molecules to adsorb onto the surface. Then the temperature was increased instantaneously from $300 \mathrm{~K}$ to $400 \mathrm{~K}$ and the system was equilibrated for $16 \mathrm{~ns}$, which allowed the unbound molecules to desorb and escape the surfactant monolayer. Subsequently the temperature was reduced back to 300 $\mathrm{K}$ for another $2 \mathrm{~ns}$. The final coverage was then estimated by determining the number of adsorbed and desorbed molecules, based on the distances of the hydroxyl oxygen atom of the headgroup to the hematite surface. ${ }^{81}$ The threshold distance $(4.0 \AA)$ was chosen such that for low concentrations, the number of adsorbed molecules at the end was equal to the number of initially placed molecules. Varying the number of the initially placed molecules allowed us to determine the maximum coverage and thus the cross-sectional area of each molecule. No base oil molecules were included in the annealing simulations since tests showed that their presence did not significantly affect the results. The same observation was made in previous MD simulations of SA monolayer stability on iron oxide surfaces. ${ }^{81}$

\section{MTT model}

Once the adsorption PMF and the area per molecule were determined, the MTT model for

surfactant adsorption developed by Nikas et al. ${ }^{35}$ was used to construct adsorption isotherms for the OFMs on hematite from PAO4. The model starts with a surface equation of state based on the following assumptions: (1) single monolayer adsorption, (2) the surfactants in the monolayer are modelled as hard-disks of radius $r$ with short-ranged attractive interactions, (3) exclusively non-ionic surfactants are considered, and (4) the headgroups are located on the Gibbs dividing surface (minimum of the PMF) while the tailgroups are pointing towards the liquid phase. From this equation of state, the surface chemical potential, $\mu^{\sigma}$, is derived.

The surfactants in the bulk are considered to be below the critical micelle concentra- 
tion (CMC), ${ }^{82}$ thus the bulk chemical potential of the surfactants $\mu^{b}$ is that of an ideal solution with no contributions coming from the interactions between surfactants. Imposing thermodynamic equilibrium $\mu^{\sigma}=\mu^{b}$, the surface coverage $\Gamma$ and the bulk molar fraction $X$ are related in a transcendental equation that can be solved using the bisection or Newton method. For more details, see the original publication ${ }^{35}$ or the derivation from Sresht et al. ${ }^{54}$ The final result reads:

$$
\ln X=\frac{\Delta \mu^{0}}{k_{B} T}+\ln \left(\frac{\Gamma a}{1-\Gamma a}\right)+\Gamma a \frac{3-2 \Gamma a}{(1-\Gamma a)^{2}}+2 B \Gamma
$$

where $X$ is the bulk mole fraction of monomer surfactant (assumed here to be the total concentration of surfactant without distinguishing between monomers, dimers, or micelles), $\Gamma$ is the surface coverage, $a$ is the hard-disk area, and $\Delta \mu^{0} \equiv \mu^{\sigma, 0}-\mu^{b, 0}$ is the free energy of adsorption at infinite dilution, i.e. with only one molecule. And $B$ is the coefficient associated to attractive pairwise interactions between surfactant molecules, which won't be taken into account in our computational results. $\Delta \mu^{0}$ is calculated from the transfer free energy or difference in free energy between the bulk region and the adsorbed state. If the interface region is broad and not well defined, then the minimum of the PMF can be used directly for $\Delta \mu^{0}$, considering that the surfactants are located at the Gibbs dividing surface, as proposed by Sresht et al. ${ }^{54}$ Here, however, we follow the approach used by Jaishankar et al., ${ }^{34}$ where the surfactants are not considered to be in a 2D plane above the hematite surface, but inside a 3D interface, where the different adsorbed states are taken into account. In this case, the expression for $\Delta \mu^{0}$ reads:

$$
\Delta \mu^{0}=-k_{B} T \ln \left[\frac{a L}{v_{s}\left(z_{1}-z_{0}\right)} \int_{z_{0}}^{z_{1}} e^{-\frac{\Delta G(z)}{k_{B} T}} d z\right]
$$

where $L=z_{2}-z_{1}, z_{2}$ marks the point at which the PMF deviates from the bulk value, $z_{1}$ is the upper bound of the free energy well and $z_{0}$ is the lower bound of the free energy well. $v_{s}$ is the molecular volume of the solvent and $\Delta G(z)$ is the adsorption PMF. 
Jaishankar et al. ${ }^{34}$ considered an additional term that takes into account the entropic penalty of increasing the coverage as the chains are not free to rotate. This contribution has been analytically estimated and increases the potential energy of the surfactants at the surface $^{83}$ and is included here as well in the MTT model:

$$
\ln X=\frac{\Delta \mu^{0}}{k_{B} T}+\ln \left(\frac{\Gamma a}{1-\Gamma a}\right)+\Gamma a \frac{3-2 \Gamma a}{(1-\Gamma a)^{2}}+2 B \Gamma+\frac{\pi^{2}}{8} N_{c} L_{c}^{4} \Gamma^{2} .
$$

Here $N_{c}=\left(n_{c}+1\right) / 3.6$ is what Nagarajan et al. ${ }^{83}$ consider as an orientationally free segment formed by 3.6 methylene groups, with $n_{c}-1$ being the number of methylene groups $\mathrm{CH}_{2}$ in the tail and the final methyl group counting as two methylenes. Each group of 3.6 methylenes is considered to be a free-orientation segment of length $L_{c}=0.46$. For a more detailed explanation of the MTT model, see Refs. 34-36.

In hydrocarbon solvents, carboxylic acids such as $\mathrm{SA}^{84}$ dimerise, while glycerol esters, such as $\mathrm{GMO}^{85}$ and $\mathrm{GMS}^{86}$ can form reverse micelles. Previous MD simulations have shown that SA monomers and dimers exhibit equal affinity for iron oxide surfaces. ${ }^{34}$ As micellation is not taken into account here, the results will be representative of systems below the CMC of the surfactants in the hydrocarbon solvent. ${ }^{82}$ To our knowledge, the CMC has not been quantified for the systems studied here. Future work could use the same techniques adopted here to study the adsorption of reverse micelles on solid surfaces. ${ }^{56,87}$

\section{Experiments}

To validate the calculations of the adsorption free energy, friction was used as a proxy for surface coverage using the Jahanmir and Beltzer model. ${ }^{20}$ The obtained surface coverage can then be related to the adsorption energy. Friction measurements were performed using a highfrequency reciprocating rig (HFRR) from PCS Instruments (Acton, UK). ${ }^{88}$ The standard specimens were used, namely a $6 \mathrm{~mm}$ diameter ball (upper) loaded on a $10 \mathrm{~mm}$ diameter disk 
(lower). Both of the specimens were made from AISI E-52100 steel. A $2 \mathrm{~N}$ load was applied to the upper specimen, which followed a reciprocating movement of $10 \mathrm{~Hz}$ with a stroke length of $0.1 \mathrm{~mm}$, giving a maximum velocity of $2 \mathrm{~mm} \mathrm{~s}^{-1}$. The corresponding maximum Hertzian pressure was $0.8 \mathrm{GPa}$, whereas the mean was $0.5 \mathrm{GPa}$. High pressure and low speed were used to ensure that boundary lubrication conditions were maintained over most of the stroke. This is important since it is here that the friction behaviour depends strongly on the OFM concentration and consequently the coverage. ${ }^{12,24}$ Ambient temperature (300 K) was used and each test was conducted for $1 \mathrm{~h}$ to reach steady state friction. The friction coefficient was measured for pure base oil and for OFM concentrations between $10^{-6} \mathrm{M}$ and $0.02 \mathrm{M}$, for a total number of $7 \mathrm{HFRR}$ tests. At the beginning of each test and once the contact was flooded, the ball and disc were held stationary and no pressure was applied during 5 minutes to allow for the formation of the OFM film, which is similar to the procedure used by Fry et al. ${ }^{25}$ The two specimens were cleaned prior to each test with acetone and toluene. The base oil used was PAO4 from ExxonMobil Chemical (Houston, USA) and the OFMs were GMO and GMS supplied by Sigma-Aldrich (Madrid, Spain) with a high purity grade (> 99\%). The steady-state friction coefficient was determined averaging over the last $10 \mathrm{~min}$ of the experiment. Three repetitions were used for each OFM and concentration to reduce the noise in the HFRR data.

To validate the calculation of the adsorption energy at infinite dilution, the Jahanmir and Beltzer model was used. ${ }^{20}$ This model relates the measured friction from the HFRR experiments with the fractional coverage. The model assumes that the contact is in boundary lubrication conditions (so friction is given by asperity contact and not the viscosity of the base oil) and that the friction coefficient is a linear function of the fractional coverage:

$$
f=f_{b}(1-\theta)+f_{a} \theta,
$$

where $f$ is the measured friction coefficient, $\theta \equiv \Gamma / \Gamma_{\max }$ is the fractional coverage, $f_{a}$ is the friction coefficient at maximum additive coverage and $f_{b}$ is the friction coefficient with 
no additive present. Although we considered $\theta=1$ to determine $f_{a}$ from the HFRR measurements, complete coverage is not achieved in practice according to our computational adsorption isotherm results. This observation is in agreement with previous MTT-MD studies. ${ }^{34}$

Once $f_{a}$ and $f_{b}$ are determined, the Jahanmir and Beltzer model can be applied to the friction data to obtain a relation between the OFM bulk concentration and the fractional surface coverage, to which an adsorption isotherm model can be fitted. Jaishankar et al. ${ }^{34}$ used the Langmuir model for this purpose. However, according to Jahanmir and Beltzer, ${ }^{20}$ the Temkin isotherm model is better suited for these OFMs than the Langmuir isotherm model since it is able to account for inhomogeneous surfaces and lateral interactions. In the Temkin isotherm model the adsorption energy increases linearly with coverage (making the adsorption process less favorable):

$$
\Delta G_{a d s}=\Delta G_{0}+\alpha \theta
$$

where $\Delta G_{0}$ is the adsorption energy at $\theta=0, \alpha$ is a positive constant depending on the interactions of the molecules on the surface, and $\theta$ refers to the fractional coverage. As explained by Jahanmir and Beltzer ${ }^{20}$ the complete form of the Temkin adsorption isotherm is complex and trying to fit several parameters to the HFRR data would result in several possible combinations of $\alpha$ and $\Delta G_{0}$. However, at intermediate fractional coverages $(0.8$ $\geq \theta \geq 0.2)$ the isotherm takes a simpler form:

$$
\theta=(R T / \alpha) \ln \left(X / K_{0}\right), K_{0}=e^{\frac{\Delta G_{0}}{R T}}
$$

Representing the fractional coverage as a function of $\ln (X), \alpha$ can be found from the slope $m$, whereas $\Delta G_{0}$ is found from the intercept $n$ as shown in equations 8 and 9. 


$$
\begin{aligned}
\alpha & =\frac{R T}{m} \\
\Delta G_{a d s} & =-\alpha n
\end{aligned}
$$

\section{Results and discussion}

\section{Adsorption}

For both solvents, the bulk density in the centre of the film was in good agreement with experimental values. We obtained approximately $742 \pm 5 \mathrm{~g} / \mathrm{L}$ for $n$-hexadecane and $778 \pm 20$ $\mathrm{g} / \mathrm{L}$ for PAO4, which is below the experimental values by $2 \%$ and $4 \%$, respectively. At the end of the equilibration phase, the separation between the two walls was $12 \mathrm{~nm}$ for $n$ hexadecane and $11 \mathrm{~nm}$ for PAO4. The interaction of the hematite surface with the base oil gave in both cases rise to a layering of the solvent molecules, as can be seen from the mass density profiles of the solvent molecules after equilibration in Fig. 3. The layering next to the wall is stronger for linear $n$-hexadecane than branched PAO4, in agreement with previous MD simulations. ${ }^{89}$ The layers are slightly thicker for PAO4 than $n$-hexadecane.

The PMF is calculated by integrating the negative of the mean force and taking its value in the bulk region as reference (i.e. zero). The bulk solvent is defined as the region where the interactions of both the OFM and the solvent with the surface are negligible (3-4 nm from the surface). For the molecules considered here, the mean force $F(\xi)$ close to the surface and thus the changes of the PMF in that region are mainly determined by the interaction of the headgroup with the surface, whereas the mean force in the transition from the bulk to the surface proximity is mainly determined by the tailgroup-solvent interactions, since the interactions of the polar headgroups with the surface are still weak. As we have chosen as reference the free energy within the bulk to be zero, the depth of the minimum will 

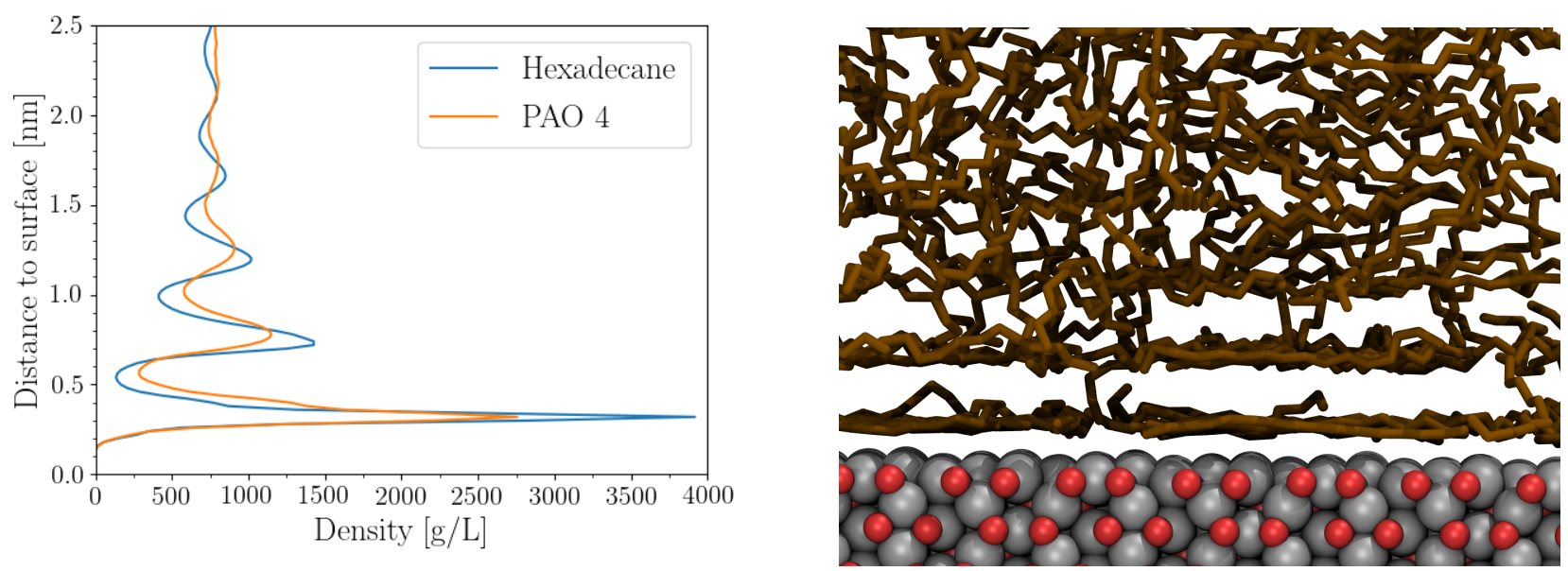

Figure 3: Mass density profiles for the solvent molecules at the end of the equilibration phase (left) and corresponding image from $\mathrm{VMD}^{60}$ for $n$-hexadecane (right). Atom colors the same as in Fig. 2.

be determined by the interactions of the OFM molecule with both the surface and the solvent.

All calculated adsorption PMFs (Figs. 4a, 4b, 4c) exhibit at least one minimum close to the surface. The PMF profiles also show energy barriers that correspond to the interaction of the tail with the layering of the solvent molecules close to the surface. There are only minor differences between PMFs from $n$-hexadecane and PAO4. The simulation time required to reach convergence of the $\mathrm{PMF}$ profile is much greater for PAO4 than $n$-hexadecane, due to its longer relaxation times. ${ }^{34}$ The results for SA are in reasonable agreement with the ones from Jaishankar et al. ${ }^{34}$ for the adsorption of $\mathrm{SA}$ from $n$-hexadecane onto $\mathrm{Fe}_{3} \mathrm{O}_{4}(111)$. As the SA molecule approaches the $\alpha-\mathrm{Fe}_{2} \mathrm{O}_{3}(010)$ surface, the free energy oscillates with a period $(\sim 0.5$ $\AA$ ) that corresponds to the thickness of the solvent layer (Fig. 3). At OFM-surface distances of around $2 \AA, 1.5 \AA, 1.0 \AA$, and $0.5 \AA$, there are free energy barriers that correspond to the OFM moving through the solvent layers. The tailgroup is preferentially located in the maxima of the solvent mass density profile, and a small energy barrier has to be crossed for the tailgroup to move between these maxima. The height of these free energy barriers increases as the OFM gets closer to the surface, because the solvent layering is more pronounced. The final barrier, corresponding to moving through the last solvent layer, is approximately 


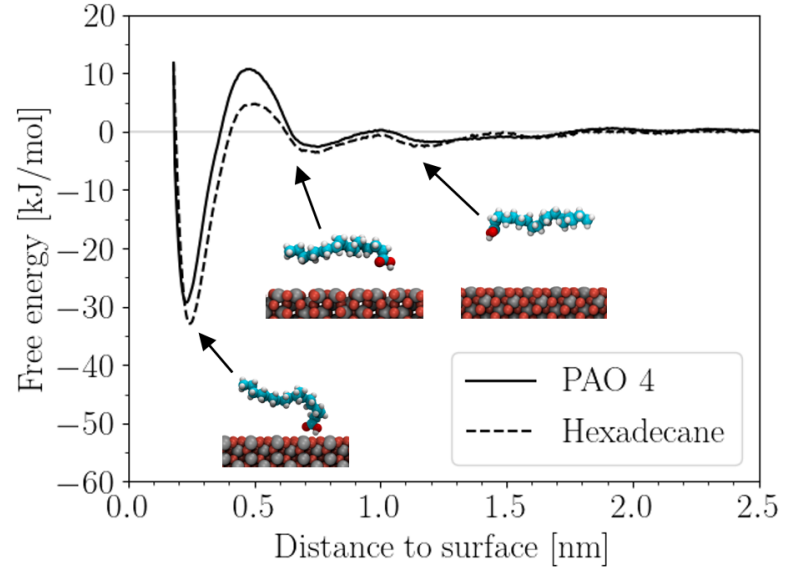

(a)

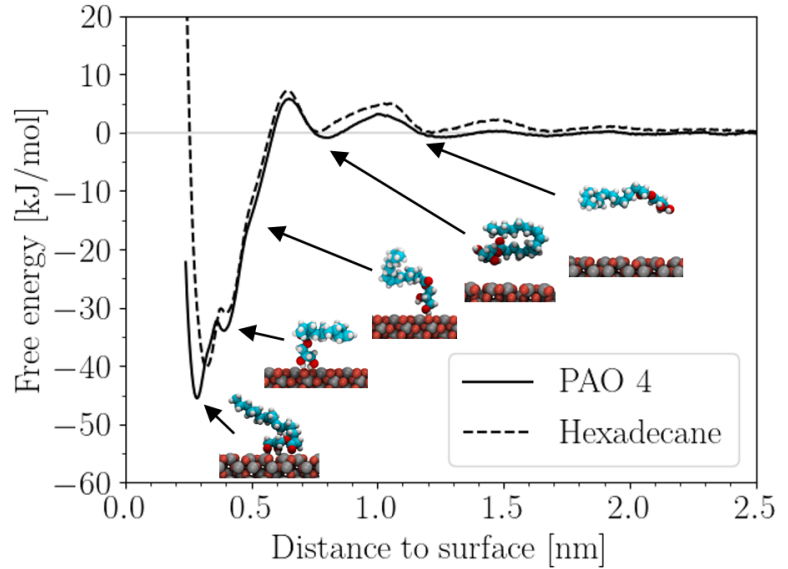

(b)

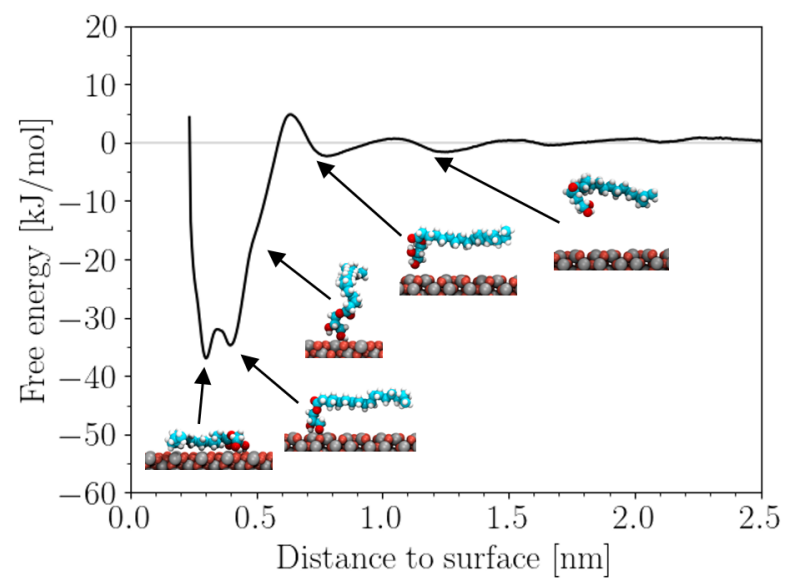

(c)

Figure 4: Adsorption PMF for the SA (a), GMO (b), and GMS (c) onto the $\alpha-\mathrm{Fe}_{2} \mathrm{O}_{3}(010)$ surface from PAO4 and $n$-hexadecane obtained using the ABF algorithm. ${ }^{42,75} \mathrm{VMD}$ snapshots ${ }^{60}$ show the molecular configurations at different points during the adsorption process from PAO4 (solvent molecules not shown for clarity). Atom colors the same as in Fig. 2. 
$10 \mathrm{~kJ} \mathrm{~mol}^{-1}$ for $\mathrm{SA}$ in PAO4 and approximately $5 \mathrm{~kJ} \mathrm{~mol}^{-1}$ for the other OFM-solvent combinations. For SA, the barrier height is larger for PAO4 than $n$-hexadecane, despite the stronger layering for the latter (Fig. 3). This implies that the solvent layers are more difficult for SA to penetrate for branched alkanes solvents than linear ones. In practical terms, this means that SA adsorption will be slower from PAO4 than $n$-hexadecane at $300 \mathrm{~K} .{ }^{34}$ The improved ability for the saturated linear OFM molecule to penetrate the $n$-alkane of similar chain length could be the molecular mechanism of the chain matching phenomena observed experimentally. ${ }^{90}$ For GMO, the free energy barrier heights are similar for both solvents. In between these barriers, there are local minima in the free energy profiles corresponding to outer-sphere complexes. These have also been observed in previous MD simulations of oxalic acid adsorption on rutile from water ${ }^{91}$ and napthenic acid adsorption on calcite from water. ${ }^{92}$

For SA, the headgroup was defined as the carboxyl group - COOH. Only one innersphere adsorption mode was observed in the PMF at $2.3 \AA$ above the surface, see Fig. 4a. The $-\mathrm{OH}$ group of the carboxylic acid was oriented towards either one oxygen atom or a bridge between two oxygen atoms of the hematite surface with a $\mathrm{OH} \cdots \mathrm{O}$ distance of $\sim 1.6$ $\AA$, while the $=\mathrm{O}$ atom of the carboxylic group was oriented towards a Fe atom of the surface with a $=\mathrm{O} \cdots$ Fe distance of $\sim 0.25 \mathrm{~nm}$. This configuration gave an adsorption energy of -27.8 $\mathrm{kJ} \mathrm{mol}^{-1}$ for $\mathrm{SA}$ on $\alpha-\mathrm{Fe}_{2} \mathrm{O}_{3}(010)$ from PAO4. The SA adsorption is slightly stronger (-32.4 $\mathrm{kJ} \mathrm{mol}^{-1}$ ) from $n$-hexadecane, because the tailgroup is only able to lie flat to the surface in the latter case. This value is in good agreement with that obtained by Jaishankar et al. ${ }^{34}$ for $\mathrm{SA}$ adsorption from $n$-hexadecane onto $\mathrm{Fe}_{3} \mathrm{O}_{4}(111)\left(29.1 \mathrm{~kJ} \mathrm{~mol}^{-1}\right)$.

For both GMO and GMS, the headgroup was defined as the carboxyl and glycerol groups $-\mathrm{COO}-\mathrm{C}_{3} \mathrm{H}_{5} \mathrm{O}_{2} \mathrm{H}_{2}$. Both of these surfactants have several polar groups capable of interacting with the oxygen or iron atoms in the surface. They are also large, flexible molecules that can adopt many different possible conformations at the solid-liquid interface. Consequently, 
two distinct inner-sphere adsorption modes are present for GMS and GMO, as can be seen from the corresponding PMFs. The different situations and adsorption modes depicted in Fig. $4 \mathrm{~b}$ and $4 \mathrm{c}$ are associated to changes in the derivative of the PMF, i.e. changes in the mean force experienced by the colvar at that distance. The barriers between $0.6 \mathrm{~nm}$ and the bulk are once again due to the interaction between the tail and the solvent molecules. At $\xi \sim 0.5 \mathrm{~nm}$, the first of the two hydroxyl groups interacts with the oxygen atoms of the hematite surface with a $\mathrm{OH} \cdots \mathrm{O}$ distance of $\sim 1.6 \AA$. At $\xi \sim 0.4 \mathrm{~nm}$, both hydroxyl groups are interacting with the oxygen atoms of the surface, and the PMF exhibits a local minimum. $\xi \sim 0.3 \mathrm{~nm}$ corresponds to the global adsorption minimum, where the $=\mathrm{O}$ atom of the headgroup also interacts with the surface with $\mathrm{a}=\mathrm{O} \cdots \mathrm{Fe}$ distance of $\sim 2.6 \AA$. There is a small energy barrier between the two adsorption modes related to the energy required to further approach the headgroup center of mass to the hematite surface. In this transition, the tailgroup goes from being located at the second maximum of the solvent mass density profile at $0.7-0.8 \mathrm{~nm}$ to being partially or completely located at the first maximum at 0.3 $\mathrm{nm}$ from the surface. In the case of GMS, the tailgroup at the global minimum lies flat to the surface, whereas for GMO, the half of the $\mathrm{C}$ atoms lie flat to the surface, with the remaining half pointing into the solvent layer due to the presence of the $Z$-alkene group. The adsorption of the alkene group provides a significant increase in adsorption strength for $\operatorname{GMO}\left(-41.8 \mathrm{~kJ} \mathrm{~mol}^{-1}\right)$ than $\operatorname{GMS}\left(-34.2 \mathrm{~kJ} \mathrm{~mol}^{-1}\right)$ on $\alpha-\mathrm{Fe}_{2} \mathrm{O}_{3}(010)$ from PAO4. This

observation is in agreement with previous experiments of fatty acids on steel surfaces ${ }^{93}$ as well as MD simulations of fatty acids on DLC surfaces. ${ }^{94}$ The adsorption energy for GMO is slightly larger from branched PAO4 than linear $n$-hexadecane $\left(-39.0 \mathrm{~kJ} \mathrm{~mol}^{-1}\right)$, which is opposite to the trend observed for SA.

\section{Hard-disk area}

The hard-disk area was determined using annealing MD simulations. ${ }^{80}$ Various monolayers of different coverages were created for the three OFMs, namely from $3.5 \mathrm{~nm}^{-2}$ to $6.5 \mathrm{~nm}^{-2}$ 
for SA and from $3.5 \mathrm{~nm}^{-2}$ to $5 \mathrm{~nm}^{-2}$ for GMO and GMS. The final coverage, or alternatively the final area per molecule, can be obtained by counting the number of molecules adsorbed at the end of the simulation. ${ }^{81}$ The threshold distance to consider a molecule as desorbed was $4 \AA$, measured from the oxygen of the hydroxyl group to the COM of the first layer of surface atoms. Increasing the threshold distance above $3.5 \AA$ did not change the results significantly, whereas decreasing it below this value underestimated the number of adsorbed molecules at low coverages, where all the molecules are assumed to be adsorbed, as shown in the Supporting Information.

Fig. 5 shows that the number of adsorbed molecules at the end of the simulation is equal to the number of molecules initially placed on the surface up to a certain value of the initial coverage. Beyond this critical value, a plateau is reached and the final coverage does not increase further regardless of the initial coverage. This maximum number of adsorbed molecules is 170 for SA, 121 for GMO and 120 for GMS. Consequently, the area per molecule at maximum coverage is $17.96 \AA^{2}$ for SA, $25.23 \AA^{2}$ for GMO and $25.44 \AA^{2}$ for GMS.

It is important to note that these results only give information regarding the minimum area due to steric effects that can be occupied by molecules adsorbed on the surface in a thermodynamically stable monolayer. It is not necessarily a realistic depiction of the maximum coverage that can be kinetically accessed in realistic timescales, since the OFM monolayers are preformed. Jaishankar et al. ${ }^{34}$ suggested that the maximum accessible coverage can be illustrated both using the MTT and the random sequential adsorption (RSA) model. ${ }^{95}$ The RSA model describes the irreversible adsorption of hard-disks on a uniform surface, which predicts a maximum coverage below the closed-packed maximum one, in which all of the surface would be covered. This is a result of irreversible adsorption and absence of restructuring between adsorption events, creating a jamming limit that was in agreement with the results by Jaishankar et al. ${ }^{34}$

In reality the value of the effective hard-disk area occupied by each molecule on the surface 


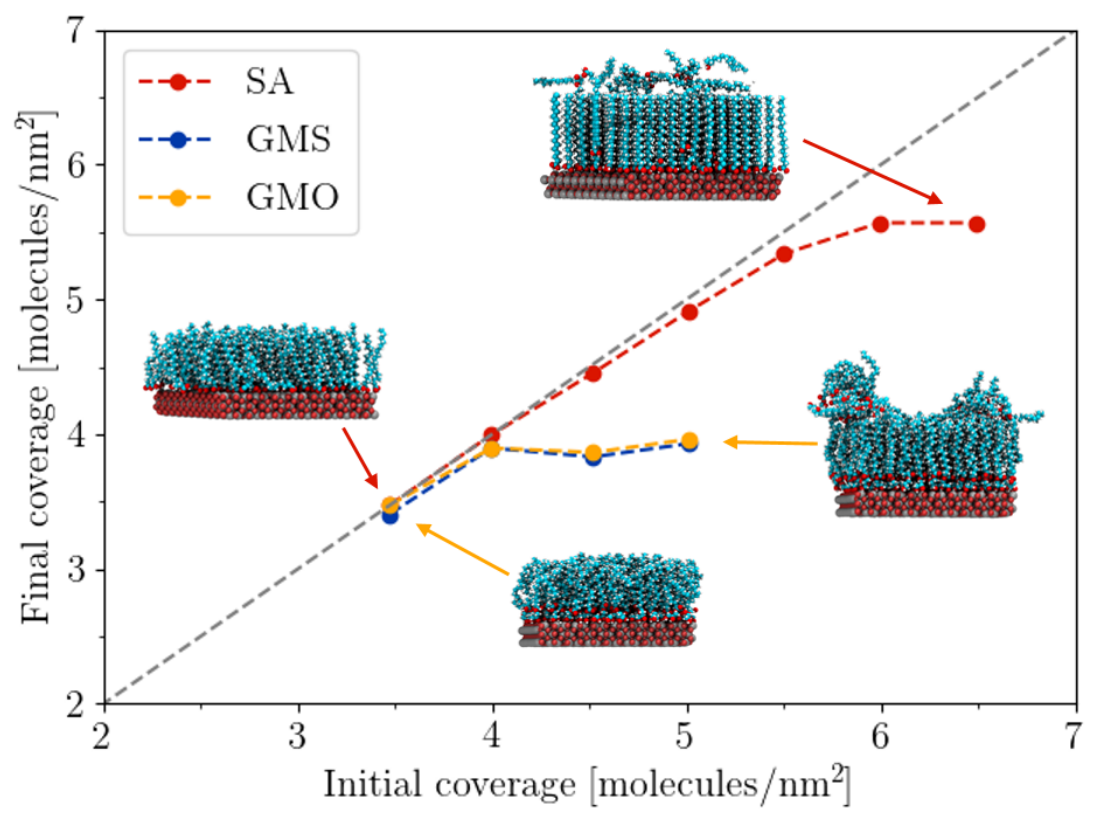

Figure 5: Comparison of the initial and final surface coverage for the three OFMs following the annealing simulations using a threshold OFM-surface distance of $4 \AA$. Colored dashed lines are guides for the eye. VMD snapshots ${ }^{60}$ show the molecular configurations after the annealing process. Atom colors the same as in Fig. 2.

depends on the coverage, in particular if the molecule has access to several adsorption modes with different conformations. The hard-disk area parameter used in the MTT model should correspond to the case of high coverage, where steric effects become important as explained by Nikas et al. ${ }^{35}$ From the literature, one can find several values for different OFMs. One should distinguish between area per molecule (total area divided by the number of molecules) and effective hard-disk area (due to steric effects, serving as input for the MTT model). The former will always be greater than or equal than the later. Davidson et al. ${ }^{55}$ calculated the area per molecule for 1-monooctyl glycerol at high coverage with MD, finding an average area per molecule of $0.225 \mathrm{~nm}^{2}$. Kawaguchi et al. ${ }^{96}$ studied the surface properties of mono-, di- and triglycerol monostearate monolayers at the air-water interface, finding a limiting area per GMS molecule of $0.26 \mathrm{~nm}^{2}$. They explain that the area per molecule of GMS is larger than the area per molecule of SA $\left(0.2 \mathrm{~nm}^{297}\right.$ or $\left.0.22 \mathrm{~nm}^{298}\right)$ due to the larger headgroup size. 
The translation from area per molecule to hard-disk area requires the disk packing efficiency to be estimated. The packing efficiency would be 0.906 for hexagonal packing (hp) or 0.785 for square packing (sp). The packing efficiency of the OFMs is likely to be tighter if there are stronger interactions between them (e.g. hydrogen bonds or van der Waals interactions between tailgroups).

Table 1: Values of the hard-disk area obtained from the annealing MD simulations on $\alpha$ $\mathrm{Fe}_{2} \mathrm{O}_{3}(010)$. $A_{\text {sim }}$ refers to the area per molecule at maximum coverage, $A_{h p}$ refers to the effective hard-disk area per molecule assuming hexagonal packing.

\begin{tabular}{ccc}
\hline OFM & $A_{\text {sim }}\left[\mathrm{nm}^{2}\right]$ & $A_{h p}\left[\mathrm{~nm}^{2}\right]$ \\
\hline SA & 0.180 & 0.163 \\
GMO & 0.252 & 0.228 \\
GMS & 0.254 & 0.230 \\
\hline
\end{tabular}

A summary of all the values for the area per molecule and the effective hard-disk area per molecule can be found in Table 1. As hexagonal packing is the most efficient way to pack disks on a surface, it is reasonable to assume at most a ratio of 0.906 between the raw area per molecule and the hard-disk area. Consequently, the results of the annealing simulations assuming hexagonal packing $\left(A_{h p}\right.$ in Table 1$)$ are then used as input for the MTT model. The values in Table 1 are in good agreement with those available in the literature for similar molecules. ${ }^{55,96-98}$ Interestingly, the estimated hard-disk area for both GMO and GMS is very similar. This is in contrast with studies from Wood et al., ${ }^{21}$ who measured a lower surface coverage for unsaturated OFMs with respect to their saturated counterparts. They explained these findings in terms of a reduced surface packing efficiency due to the presence of the kinked $Z$-alkene. However, in the annealing simulations presented here no significant difference is observed between both, suggesting that the observed differences in surface coverage by Wood et al. ${ }^{21}$ are instead due to kinetic effects due to steric hindrance, rather than surface packing efficiency. 


\section{Adsorption isotherms from MTT}

The main input parameters for the MTT model are the adsorption energy, the hard-disk area, and the number of orientationally-free carbon atoms $n_{c}$ in the tail. The value of the harddisk area for each OFM is taken as $A_{h p}$ from Table 1. The adsorption energy is calculated for each OFM using equation 3 and the PMF, with the solvent volume $v_{s}$ for PAO4 being $0.8525 \mathrm{~nm}^{3}$ and $0.488 \mathrm{~nm}^{3}$ for Hexadecane. For SA the adsorption energy was calculated using $z_{0}=0.18 \mathrm{~nm}, z_{1}=0.365 \mathrm{~nm}$ and $z_{2}=1.8 \mathrm{~nm}$ for PAO4 and yielded $\Delta \mu_{s a}^{0}=-27.8$ $\mathrm{kJ} \mathrm{mol}{ }^{-1}$. While for the Hexadecane results $z_{0}=0.18 \mathrm{~nm}, z_{1}=0.4 \mathrm{~nm}$ and $z_{2}=1.8$ which yielded $\Delta \mu_{s a}^{0}=-32.4 \mathrm{~kJ} \mathrm{~mol}^{-1}$. For the adsorption of GMO from PAO4, we used $z_{0}=0.22$ $\mathrm{nm}, z_{1}=0.59 \mathrm{~nm}$ and $z_{2}=1.4 \mathrm{~nm}$ and obtained an adsorption energy $\Delta \mu_{g m o}^{0}=-41.8 \mathrm{~kJ}$ $\mathrm{mol}^{-1}$. Whereas for the corresponding Hexadecane results $z_{0}=0.25 \mathrm{~nm}, z_{1}=0.57 \mathrm{~nm}$ and $z_{2}=1.63$ which yielded $\Delta \mu_{\text {gmo }}^{0}=-39.0 \mathrm{~kJ} \mathrm{~mol}^{-1}$. For GMS the same values of $z_{0}, z_{1}$ and $z_{2}$ are used as in GMO-PAO4 and the adsorption energy is $\Delta \mu_{g m s}^{0}=-34.2 \mathrm{~kJ} \mathrm{~mol}^{-1}$. The value of $n_{c}$ was taken as 17 for SA and GMS and 15 for GMO, since the $\mathrm{C}$ atoms involved in the alkene group are not counted.

The adsorption isotherm for SA calculated with MTT (Eq. (4)) ${ }^{35}$ with parameters obtained from the MD simulations ${ }^{34,36}$ is compared to data from previous iron oxide powder adsorption experiments in Fig. 7. The adsorption isotherm without accounting for lateral interactions between adsorbed surfactants $(B=0)$, a simplification suggested by Jaishankar et al., ${ }^{34}$ is shown as a solid line. Using this approach, the MTT results are in good agreement with the experimental results reported by Jaishankar et al. ${ }^{34}$ from $n$-hexadecane. The surface coverage at intermediate concentrations is underestimated compared to the experimental results from Wood et al. ${ }^{21}$ from $n$-dodecane and Studt ${ }^{99}$ from $n$-hexadecane. The calculated adsorption free energy from the PMF of SA on $\alpha-\mathrm{Fe}_{2} \mathrm{O}_{3}(010)$ from $n$-hexadecane $\left(-32.4 \mathrm{~kJ} \mathrm{~mol}^{-1}\right)$ agrees well with previous estimates $\left(-29.1 \mathrm{~kJ} \mathrm{~mol}^{-1}\right)$ on $\mathrm{Fe}_{3} \mathrm{O}_{4}(111)$ from

$n$-hexadecane. ${ }^{34}$ Moreover, the hard-disk area (Table 1) is also consistent with previous estimates of the molecular area of SA. ${ }^{97,98}$ 
Consequently, we suggest that the rate at which coverage increases with OFM concentration is underestimated at intermediate coverage compared to some experiments due to the absence of lateral interactions in this version of the MTT model. To account for these interactions, one should estimate the dispersion forces between adsorbed molecules on the surface and then calculate the $B$ parameter of Eq. (4), as explained in Refs. 35,36. One possibility would be to calculate the PMF between two adsorbed OFMs using the distance between headgroups as colvar, as suggested by Sresht et al. ${ }^{36}$ These calculations are reported for our systems in the Supporting Information. Our results yield an overestimation of the $B$ parameter, which we attribute to issues with convergence of the PMF profiles. To obtain satisfactory convergence for these systems would require several $\mu$ s of simulation time. However, to show that including these lateral interactions would approach our results to the experimental ones, we have used a non-linear least squares fitting of Eq. (4) to the experimental data, using the same values for the hard-disk area and the adsorption energy as before, while optimizing $B$ (see Fig. 7). The resulting MTT adsorption isotherms closely match the experimental data where $B=0.114 \mathrm{~nm}^{2}$ for Jaishankar et al., ${ }^{34} B=-0.453 \mathrm{~nm}^{2}$ for Wood et al., ${ }^{21}$ and $B=-0.847 \mathrm{~nm}^{2}$ for Studt. ${ }^{99}$ A negative $B$ value is expected due to the attractive nature of the interactions. The values obtained to fit the experimental data are similar in magnitude to those obtained previously for $\mathrm{C}_{10} \mathrm{E}_{4}$ polyethylene glycol surfactants at the air-water interface. ${ }^{36}$ The accurate determination of lateral interactions for the OFMs studied here will be the focus of future MD simulations. This will likely require the use of a smaller hydrocarbon solvent to accelerate convergence and modification of the colvar to accelerate the exploration of the different degrees of freedom, such as the tailgroup positions with respect to the headroups. 


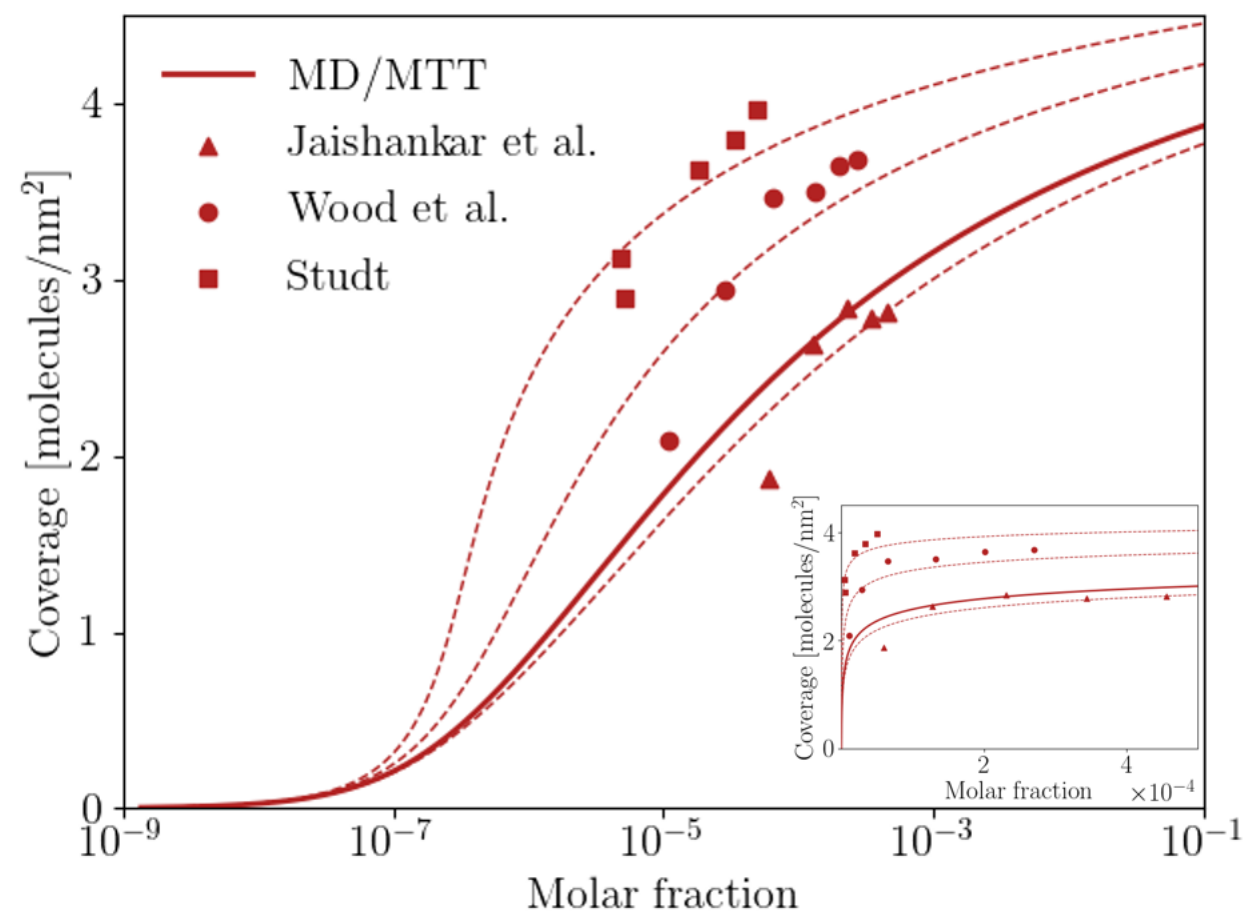

Figure 6: Adsorption isotherm obtained using the MTT model (Eq. (4) $)^{35}$ for SA on $\alpha$ $\mathrm{Fe}_{2} \mathrm{O}_{3}(010)$ from $n$-hexadecane, with the parameters calculated from the MD simulations (solid line). The results are compared to previously obtained experimental data (points) for SA adsorption on iron oxide powder from $n$-dodecanol (Wood et al. ${ }^{21}$ ) and $n$-hexadecane (Jaishankar et al. ${ }^{34}$ and Studt ${ }^{99}$ ). Fits of the MTT model including lateral interactions ( $B$ parameter in Eq. (4)) are also included for each experimental data-set for SA (dashed lines).

Fig. 7 shows adsorption isotherms obtained using the MTT model (Eq. (4) ${ }^{35}$ for the three OFMs on $\alpha-\mathrm{Fe}_{2} \mathrm{O}_{3}(010)$ from PAO4, with the parameters calculated from the MD simulations, without lateral interactions $(B=0)$. Of the three OFMs studied, SA yields the highest maximum coverage $\left(3.6 \mathrm{~nm}^{-2}\right)$ due to its smaller headgroup size compared to GMO and GMS. This is in agreement with the maximum coverage of SA obtained experimentally by Wood et al. ${ }^{21}$ and Studt, ${ }^{99}$ validating the value used for the hard-disk area. Since both GMO and GMS have the same headgroup, they yield a very similar maximum coverage at high concentrations, where the surface coverage is mostly determined by the packing efficiency of the molecules. GMO yields the highest coverage of the three OFMs at low concentration, which is due to its larger adsorption energy. GMS has a coverage between GMO and SA at low concentration because of its intermediate adsorption energy. 


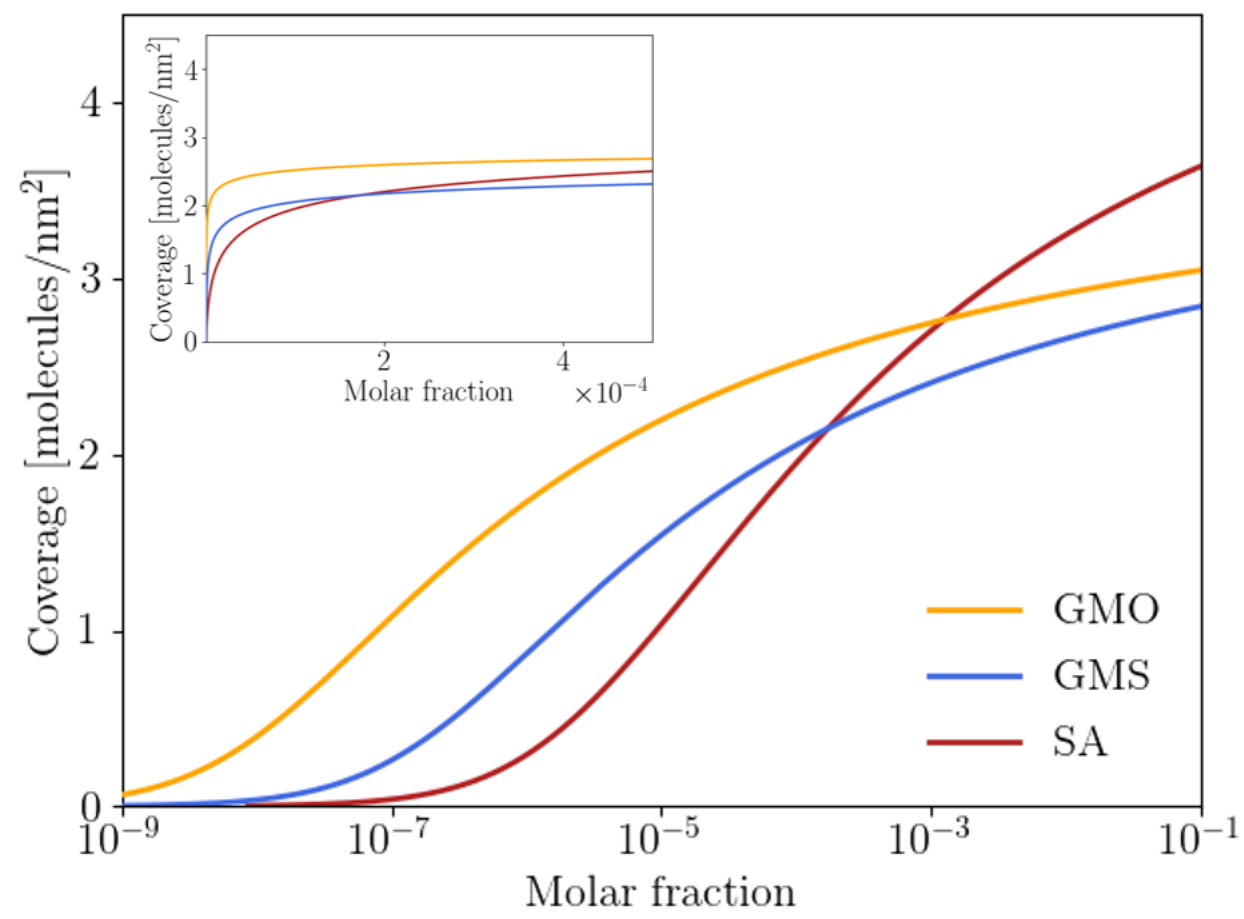

Figure 7: Adsorption isotherms obtained using the MTT model (Eq. (4)) ${ }^{35}$ for the three OFMs on $\alpha-\mathrm{Fe}_{2} \mathrm{O}_{3}(010)$ from PAO4, with the parameters calculated from the MD simulations, without lateral interactions $(B=0)$.

Experimentally, Wood et al. ${ }^{21}$ observed a higher surface coverage for fatty acids with fewer $Z$-unsaturated groups (stearic $>$ oleic $>$ linoleic) at equal concentration. Campen et al. ${ }^{24}$ showed that unsaturated OFMs gave higher friction that was independent of the sliding speed, which can be explained in terms of a low surface coverage. At equal surface coverage Ewen et al. ${ }^{66}$ found similar friction for both saturated and unsaturated fatty acids in NEMD simulations, ascribing the differences found experimentally to the superior ability of saturated OFMs to form high-coverage monolayers with respect to their unsaturated counterparts. This difference between saturated and unsaturated OFMs is not reproduced with the present methodology. The form of the MTT model that has been used here only depends on the hard-disk area and the adsorption energy. The former is very similar for both GMS and GMO, since it is mainly determined by the headgroup, while the latter is 
more negative for GMO, due to its additional $Z$-alkene adsorption site.

The version of the MTT model used here does not appear to fully account for the differences in tailgroup saturation observed experimentally. The original MTT model included an extra term (with another parameter) that took into account the van der Waals interactions between surfactants on the surface. This parameter might be able to capture the differences in tailgroup saturation.

In order to differentiate GMO and GMS (and more generally saturated and unsaturated OFMs) in the current MD-MTT framework, a slightly different approach is required. We suggest that the PMF for OFM adsorption should be obtained on iron oxide surfaces partially covered by preformed monolayers with different surface coverages. This type of simulation has been performed previously for alkyl phosphonate monolayers on aluminium oxide surfaces ${ }^{100}$ This way, the steric barriers to the formation of high-coverage monolayers could be uncovered. This, however, is likely to require extensive computational resources (several $\mu \mathrm{s}$ of simulation time) and is beyond the scope of this current study.

\section{Friction experiments}

The experimental HFRR results are obtained calculating the steady-state friction coefficient from three repetitions of the HFRR experiments at each OFM concentration. The results for GMO and GMS are shown in Figure 8. The highest friction coefficient (0.263) is observed for the pure base oil (not depicted in Figure 8). As expected, the lowest friction coefficient is observed for the highest OFM concentrations, corresponding to $0.02 \mathrm{M}$ for GMO (0.0103 in molar fraction) or $0.01 \mathrm{M}$ for GMS (0.005 in molar fraction). These concentrations correspond to the high coverage regime according to the calculated MTT adsorption isotherms (see Fig. 7), with 2-3 $\mathrm{nm}^{-2}$. This is in contrast to the results of Jaishankar et al. ${ }^{34}$ for SA.

Subsequently, the Jahanmir and Beltzer model ${ }^{20}$ is applied to the friction coefficient results to obtain the coverage as a function of the logarithm of the molar concentration. Fitting the Temkin adsorption isotherm to intermediate concentrations for each OFM, we 

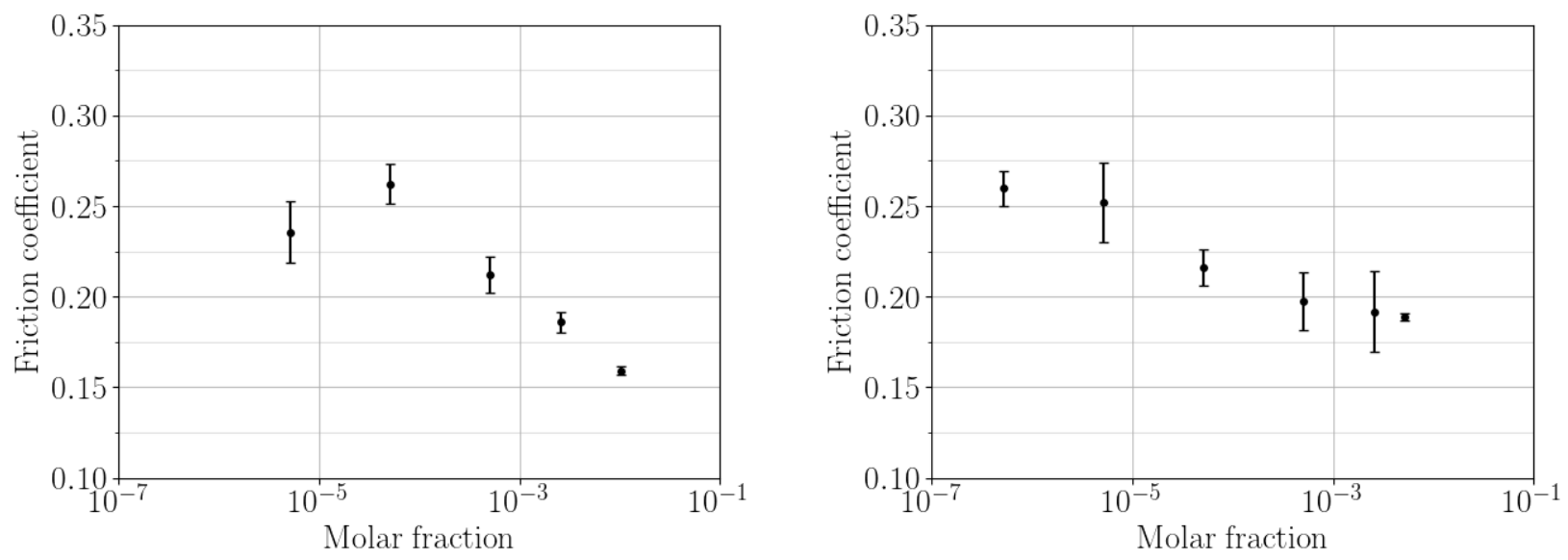

Figure 8: Steady state friction coefficient from the HFRR experiments as a function of molar concentration for GMO (left) and GMS (right). The error bars represent the standard deviation of the data used to calculate the steady-state friction coefficient.

obtain an experimental estimate of the adsorption energy, see Figure 9. This approach yields and adsorption energy of $-24.9 \mathrm{~kJ} \mathrm{~mol}^{-1}$ for GMO $(\alpha=13.1)$ and $-33.2 \mathrm{~kJ} \mathrm{~mol}^{-1}$ for GMS $(\alpha=15.5)$. These values are the same order of magnitude as the computational results calculated through the adsorption PMF of each OFM, see Table 2.

Table 2: Adsorption energy of OFMs from PAO4 or Hexadecane on ABF-MD $\left(\Delta \mu_{M D}\right)$ and experimental values for GMO and GMS estimated using fits of the Temkin isotherm to HFRR friction data on steel in PAO4 $\left(\Delta \mu_{\text {exp }}\right)$, * experimental data for SA taken from HFRR experiments in PAO6 from Ref. 34 using the Langmuir isotherm.

\begin{tabular}{cccc}
\hline OFM & SA & GMS & GMO \\
\hline$\Delta \mu_{M D}\left[\mathrm{~kJ} \mathrm{~mol}^{-1}\right]$ & -32.4 & - & -39.0 \\
$\begin{array}{c}(\text { Hexadecane }) \\
\Delta \mu_{M D}\left[\mathrm{~kJ} \mathrm{~mol}^{-1}\right]\end{array}$ & -27.8 & -34.2 & -41.8 \\
$\begin{array}{c}(\mathrm{PAO} 4) \\
\left.\Delta \mu_{\exp }[\mathrm{kJ} \mathrm{mol})^{-1}\right]\end{array}$ & $-26.4^{*}$ & -33.2 & -24.9 \\
\hline PAO4 $)$ & & & \\
\hline
\end{tabular}

The agreement between the computational and experimental results is much better for GMS than for GMO and the $\alpha$ value found for both OFMs is the same. One would expect to find different $\alpha$ values if different chain packing efficiencies lead to reduced dispersion forces between adsorbed molecules in the case of GMO and thus lower coverage. This was the case with SA and isosearic acid reported by Jahanmir and Beltzer, ${ }^{20}$ in which the presence of 
the methyl side group decreases packing efficiency and thus dispersion forces. They found a different slope $m$ and therefore a different $\alpha$ for both surfactants. The same authors, however, ascribed the differences in coverage between SA, elaidic acid and oleic acid to changes in chain dispersion interaction even though they found similar values of $\alpha$ for the three surfactants.

Overall, the differences between our computational adsorption energy (with a single molecule) and the experimental estimation fitting the Temkin adsorption isotherm for GMO can be understood through the steric hindrance for molecules adsorbing into partially formed monolayers. The kink in the $Z$-unsaturated tailgroup of GMO will lead to larger kinetic barriers for molecules to pass through the film and adsorb on the hematite surface. As well as affecting the kinetics of adsorption, this may also influence the thermodynamics. The steric barrier could also lead to a reduction in the adsorption energy for GMO (as predicted from the HFRR experiments) since on average, molecules are trapped further from the surface. Another factor worth bearing in mind is that the PMF calculation is completely agnostic to both the interaction between molecules in solution. It is possible that reverse micelle formation is more favourable in GMO than GMS, ${ }^{85,86}$ reducing the availability of molecules to adsorb on the hematite surface.
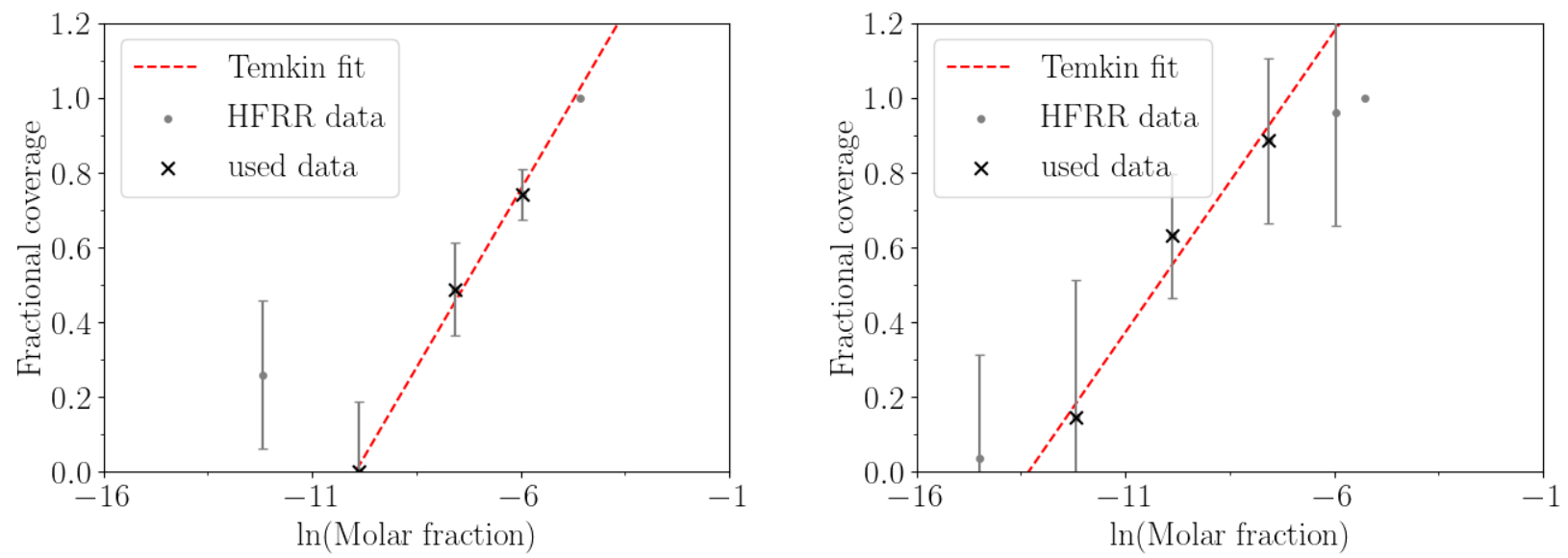

Figure 9: Fractional surface coverage as a function of the logarithm of the molar fraction with GMO (left) and GMS (right). Error bars are calculated from the propagation of the standard deviation of the friction measures. 
For the adsorption energy of SA, several references can be found in the literature. Jahanmir and Beltzer ${ }^{20}$ used their model and the Temkin adsorption isotherm as done here to estimate the adsorption energy at full coverage $\left(\Delta G_{a d s}(\theta=1)\right)$ obtaining $-23 \mathrm{~kJ} \mathrm{~mol}^{-1}$. Jaishankar et al. ${ }^{34}$ estimated the adsorption energy of SA at infinite dilution computationally, as done here through the PMF of adsorption $\left(\Delta G_{0}=-26.4 \mathrm{~kJ} \mathrm{~mol}^{-1}\right)$ and experimentally with powder adsorption experiments, quartz crystal microbalance and HFRR as done here, obtaining estimates between $-26.4 \mathrm{~kJ} \mathrm{~mol}^{-1}$ and $-29.1 \mathrm{~kJ} \mathrm{~mol}^{-1}$. These values are in excellent agreement with the computational results presented here, see Table 2.

\section{Conclusions}

In this work, we have used MD simulations together with the MTT model to predict the adsorption isotherm of three OFMs in hydrocarbon solvents onto an iron oxide surface. First, we calculated the adsorption PMF for the three OFMs using MD and the ABF method, which proved to be more efficient than other comparable enhanced sampling approaches. The computational results showed that the adsorption strength increased in the order $\mathrm{SA}<$ GMS $<$ GMO, suggesting that the adsorption energy increases with the number of functional groups that interact with the surface. The stronger adsorption energy for GMO than GMS is due to the additional $Z$-alkene function group in the tailgroup. The different characteristics of the PMF have been interpreted in terms of headgroup-surface and tailgroup-solvent interactions. Both $n$-hexadecane and PAO4 give similar results, with the calculations using PAO4 or GMS taking longer to converge, due to slower transitions of the OFM tailgroup between solvent layers. Subsequently, the hard-disk surface area has been estimated using annealing simulations of pre-formed monolayer films at different surface coverages. The results show that the hard-disk area increases in the order: SA $<$ GMS $\sim$ GMO. The adsorption energy and hard-disk area are used to calculate the adsorption isotherm using the MTT model. The amount adsorbed at low coverage is mainly controlled by the adsorption energy and the 
maximum coverage by the hard-disk area. Lateral interactions between adsorbed molecules control how fast the adsorption isotherm reaches its asymptotic value. While one experimental data-set for SA can be adequately described with only the adsorption energy and hard-disk area, two other data-sets require the lateral interactions to be accounted for. The adsorption isotherm for GMS and GMO are similar, in contrast with previous experimental results regarding the packing of saturated and unsaturated surfactants. We propose that these differences in coverage that are found experimentally, are mainly due to differences in the kinetic barriers to the formation of high-coverage monolayers, with differences in aggregation in non-polar solvents and differences in lateral interactions playing a secondary role. These factors could be investigated in future work. Finally, the calculated adsorption energies from the PMFs have been validated through HFRR experiments and the Jahanmir and Beltzer model. A good agreement is obtained between the computational results and the experimental estimation of the adsorption energy for the OFMs (SA and GMS) with saturated tailgroups, while the adsorption energy is overestimated by around $10 \mathrm{KJ} \mathrm{mol}^{-1}$ for the OFM with a $Z$-unsaturated tailgroup (GMO). We have shown that it is possible to predict the adsorption isotherm of saturated OFMs in silico. It is expected that the differences for the unsaturated OFMs can be accounted for by accounting for the decrease in adsorption energy on already partially formed monolayers.

\section{Acknowledgement}

All authors thank the Repsol Foundation and the Repsol Technology Lab for financial support. We acknowledge the Partnership for Advanced Computing in Europe (PRACE) scheme for awarding us access to the Jülich Wizard for European Leadership Science (JUWELS) system, Gauss Centre for Supercomputing at the Forschungszentrum Jülich (GCS@FZJ), Germany. ${ }^{101}$ J.P.E. was supported by the Royal Academy of Engineering under the Research Fellowship scheme. 


\section{Supporting Information Available}

Convergence of ABF calculations and second approach to hard-disk area calculations.

\section{References}

(1) Manne, S.; Gaub, H. E. Molecular Organization of Surfactants at Solid-Liquid Interfaces. Science 1995, 270, 1480-1482.

(2) McFadden, C.; Soto, C.; Spencer, N. D. Adsorption and surface chemistry in tribology. Tribology International 1998, 30, 881-888.

(3) Spikes, H. A. Direct Observation of Boundary Layers. Langmuir 1996, 12, 4567-4573.

(4) Grossiord, C.; Varlot, K.; Martin, J.-M.; Le Mogne, T.; Esnouf, C.; Inoue, K. MoS2 single sheet lubrication by molybdenum dithiocarbamate. Tribology International 1998, 31, 737-743.

(5) Jiang, S. Y.; Frazier, R.; Yamaguchi, E. S.; Blanco, M.; Dasgupta, S.; Zhou, Y. H.; Cagin, T.; Tang, Y. C.; Goddard, W. A. The SAM model for wear inhibitor performance of dithiophosphates on iron oxide. Journal of Physical Chemistry B 1997, 101, 7702-7709.

(6) Spikes, H. The History and Mechanisms of ZDDP. Tribology Letters 2004, 17, 469489.

(7) Taylor, R. I. Tribology and energy efficiency: from molecules to lubricated contacts to complete machines. Faraday Discuss. 2012, 156, 361-382.

(8) Spikes, H. A. Additive-additive and additive-surface interactions in lubrication. Lubrication Science 1989, 2, 3-23. 
(9) Rounds, F. G. Additive Interactions and Their Effect on the Performance of a Zinc Dialkyl Dithiophosphate. ASLE Transactions 1978, 21, 91-101.

(10) Zhang, J.; Yamaguchi, E.; Spikes, H. The Antagonism between Succinimide Dispersants and a Secondary Zinc Dialkyl Dithiophosphate. Tribology Transactions 2014, $57,57-65$.

(11) Guegan, J.; Southby, M.; Spikes, H. Friction Modifier Additives, Synergies and Antagonisms. Tribology Letters 2019, 67, 1-12.

(12) Spikes, H. Friction Modifier Additives. Tribology Letters 2015, 60, 1-26.

(13) Hardy, W. B.; Doubleday, I. Boundary lubrication.-The paraffin series. Proceedings of the Royal Society of London. Series A. Mathematical and Physical Sciences 1922, $100,550-574$.

(14) Bowden, F.; Leben, L. The Friction of Lubricated Metals. Philosophical Transactions of the Royal Society of London. Series A. Mathematical and Physical Sciences 1940, 239, 1-27.

(15) Fowkes, F. M. Orientation Potentials of Monolayers Adsorbed at the Metal-Oil interface. The Journal of Physical Chemistry 1960, 64, 726-728.

(16) Groszek, A. J. Heat of Preferential Adsorption of Surfactants on Porous Solids and Its Relation to Wear of Sliding Steel Surfaces. ASLE Transactions 1962, 5, 105-114.

(17) Rowe, C. N. Role of Additive Adsorption in the Mitigation of Wear. ASLE Transactions 1970, 13, 179-188.

(18) Spikes, H. A.; Cameron, A. A comparison of adsorption and boundary lubricant failure. Proceedings of the Royal Society of London. Series A. Mathematical and Physical Sciences 1974, 336, 407-419. 
(19) Jahanmir, S.; Beltzer, M. Effect of Additive Molecular Structure on Friction Coefficient and Adsorption. Journal of Tribology 1986, 108, 109-116.

(20) Jahanmir, S.; Beltzer, M. An adsorption model for friction in boundary lubrication. ASLE Transactions 1986, 29, 423-430.

(21) Wood, M. H.; Casford, M. T.; Steitz, R.; Zarbakhsh, A.; Welbourn, R. J.; Clarke, S. M. Comparative Adsorption of Saturated and Unsaturated Fatty Acids at the Iron Oxide/Oil Interface. Langmuir 2016, 32, 534-540.

(22) Ruths, M.; Lundgren, S.; Danerlov, K.; Persson, K. Friction of fatty acids in nanometer-sized contacts of different adhesive strength. Langmuir 2008, 24, 15091516.

(23) Lundgren, S. M.; Ruths, M.; Danerlöv, K.; Persson, K. Effects of unsaturation on film structure and friction of fatty acids in a model base oil. Journal of Colloid and Interface Science 2008, 326, 530-536.

(24) Campen, S.; Green, J.; Lamb, G.; Atkinson, D.; Spikes, H. On the increase in boundary friction with sliding speed. Tribology Letters 2012, 48, 237-248.

(25) Fry, B. M.; Moody, G.; Spikes, H. A.; Wong, J. S. Adsorption of Organic Friction Modifier Additives. Langmuir 2020, 36, 1147-1155.

(26) Ewen, J. P.; Heyes, D. M.; Dini, D. Advances in nonequilibrium molecular dynamics simulations of lubricants and additives. Friction 2018, 6, 349-386.

(27) Apóstolo, R. F.; Tsagkaropoulou, G.; Camp, P. J. Molecular adsorption, self-assembly, and friction in lubricants. Journal of Molecular Liquids 2019, 27r7, 606-612.

(28) De Barros-Bouchet, M. I.; Righi, M. C.; Philippon, D.; Mambingo-Doumbe, S.; LeMogne, T.; Martin, J. M.; Bouffet, A. Tribochemistry of phosphorus additives: Experiments and first-principles calculations. RSC Advances 2015, 5, 49270-49279. 
(29) Gattinoni, C.; Ewen, J. P.; Dini, D. Adsorption of surfactants on $\alpha-\mathrm{Fe}_{2} \mathrm{O}_{3}(0001)$ : a Density Functional Theory study. Journal of Physical Chemistry C 2018, 122, 2081720826.

(30) Blanck, S.; Loehlé, S.; Steinmann, S. N.; Michel, C. Adhesion of lubricant on aluminium through adsorption of additive head-groups on $\gamma$-alumina: A DFT study. Tribology International 2020, 145, 106140.

(31) Zhu, B. Y.; Gu, T. Surfactant adsorption at solid-liquid interfaces. Advances in Colloid and Interface Science 1991, 37, 1-32.

(32) Gaberle, J.; Gao, D. Z.; Watkins, M. B.; Shluger, A. L. Calculating the Entropy Loss on Adsorption of Organic Molecules at Insulating Surfaces. Journal of Physical Chemistry C 2016, 120, 3913-3921.

(33) Chia, C. L.; Alloway, R. M.; Jephson, I.; Clarke, S. M.; Filip, S. V.; Siperstein, F. R.; Avendaño, C. Competitive Adsorption of a Multifunctional Amine and Phenol Surfactant with Ethanol on Hematite from Nonaqueous Solution. Journal of Physical Chemistry B 2019, 123, 1375-1383.

(34) Jaishankar, A.; Jusufi, A.; Vreeland, J. L.; Deighton, S.; Pellettiere, J.; Schilowitz, A. M. Adsorption of Stearic Acid at the Iron Oxide/Oil Interface: Theory, Experiments, and Modeling. Langmuir 2019, 35, 2033-2046.

(35) Nikas, Y. J.; Puvvada, S.; Blankschtein, D. Surface Tensions of Aqueous Nonionic Surfactant Mixtures. Langmuir 1992, 8, 2680-2689.

(36) Sresht, V.; Lewandowski, E. P.; Blankschtein, D.; Jusufi, A. Combined Molecular Dynamics Simulation-Molecular-Thermodynamic Theory Framework for Predicting Surface Tensions. Langmuir 2017, 33, 8319-8329. 
(37) Yoon, J.; Ulissi, Z. W. Capturing Structural Transitions in Surfactant Adsorption Isotherms at Solid/Solution Interfaces. Langmuir 2020, 36, 819-826.

(38) De Angelis, P.; Cardellini, A.; Asinari, P. Exploring the Free Energy Landscape To Predict the Surfactant Adsorption Isotherm at the Nanoparticle-Water Interface. ACS Central Science 2019, 5, 1804-1812.

(39) Jusufi, A.; Jaishankar, A.; Onodera, K.; Vreeland, J.; Konicek, A. R.; Watanabe, H.; Sato, T.; Manabe, K.; Yamamori, K.; Schilowitz, A. M. Adsorption properties of molybdenum based FMs on boron-doped DLC. Wear 2019, 426-427, 805-812.

(40) Kumar, S.; Rosenberg, J. M.; Bouzida, D.; Swendsen, R. H.; Kollman, P. A. The weighted histogram analysis method for free-energy calculations on biomolecules. I. The method. Journal of Computational Chemistry 1992, 13, 1011-1021.

(41) Gallicchio, E.; Andrec, M.; Felts, A. K.; Levy, R. M. Temperature weighted histogram analysis method, replica exchange, and transition paths. Journal of Physical Chemistry B 2005, 109, 6722-6731.

(42) Darve, E.; Rodríguez-Gómez, D.; Pohorille, A. Adaptive biasing force method for scalar and vector free energy calculations. Journal of Chemical Physics 2008, 128.

(43) Henin, J.; Fiorin, G.; Chipot, C.; Klein, M. L. Exploring multidimensional free energy landscapes using time-dependent biases on collective variables. Journal of Chemical Theory and Computation 2010, 6, 35-47.

(44) Wu, M.; He, J.; Ren, X.; Cai, W.-S.; Fang, Y.-C.; Feng, X.-Z. Development of functional biointerfaces by surface modification of polydimethylsiloxane with bioactive chlorogenic acid. Colloids and Surfaces B: Biointerfaces 2014, 116, 700-706.

(45) Goel, K.; Zuñiga-Bustos, M.; Lazurko, C.; Jacques, E.; Galaz-Araya, C.; ValenzuelaHenriquez, F.; Pacioni, N. L.; Couture, J.-F.; Poblete, H.; Alarcon, E. I. Nanoparticle 
Concentration vs Surface Area in the Interaction of Thiol-Containing Molecules: Toward a Rational Nanoarchitectural Design of Hybrid Materials. ACS Applied Materials ES Interfaces 2019, 11, 17697-17705.

(46) Kuang, Z.; Singh, K. M.; Oliver, D. J.; Dennis, P. B.; Perry, C. C.; Naik, R. R. Gamma estimator of Jarzynski equality for recovering binding energies from noisy dynamic data sets. Nature Communications 2020, 11, 5517.

(47) Friddle, R. W.; Battle, K.; Trubetskoy, V.; Tao, J.; Salter, E. A.; Moradian-Oldak, J.; De Yoreo, J. J.; Wierzbicki, A. Single-molecule determination of the face-specific adsorption of Amelogenin's C-terminus on hydroxyapatite. Angewandte Chemie International Edition 2011, 50, 7541-7545.

(48) Poblete, H.; Miranda-Carvajal, I.; Comer, J. Determinants of Alanine Dipeptide Conformational Equilibria on Graphene and Hydroxylated Derivatives. Journal of Physical Chemistry B 2017, 121, 3895-3907.

(49) Olsen, R.; Leirvik, K. N.; Kvamme, B.; Kuznetsova, T. Adsorption Properties of Triethylene Glycol on a Hydrated \{1014\} Calcite Surface and Its Effect on Adsorbed Water. Langmuir 2015, 31, 8606-8617.

(50) Olsen, R.; Leirvik, K. N.; Kvamme, B. Adsorption characteristics of glycols on calcite and hematite. AIChE Journal 2019, 65, e16728.

(51) Comer, J.; Chen, R.; Poblete, H.; Vergara-Jaque, A.; Riviere, J. E. Predicting Adsorption Affinities of Small Molecules on Carbon Nanotubes Using Molecular Dynamics Simulation. ACS Nano 2015, 9, 11761-11774.

(52) Azhagiya Singam, E. R.; Zhang, Y.; Magnin, G.; Miranda-Carvajal, I.; Coates, L.; Thakkar, R.; Poblete, H.; Comer, J. Thermodynamics of Adsorption on Graphenic Surfaces from Aqueous Solution. Journal of Chemical Theory and Computation 2019, 15, 1302-1316. 
(53) Wang, Y.; Comer, J.; Chen, Z.; Chen, J.; Gumbart, J. C. Exploring adsorption of neutral aromatic pollutants onto graphene nanomaterials via molecular dynamics simulations and theoretical linear solvation energy relationships. Environmental Science: Nano 2018, 5, 2117-2128.

(54) Sresht, V.; Lewandowski, E. P.; Blankschtein, D.; Jusufi, A. Combined Molecular Dynamics Simulation-Molecular-Thermodynamic Theory Framework for Predicting Surface Tensions. Langmuir 2017, 33, 8319-8329.

(55) Davidson, J. E.; Hinchley, S. L.; Harris, S. G.; Parkin, A.; Parsons, S.; Tasker, P. A. Molecular dynamics simulations to aid the rational design of organic friction modifiers. Journal of Molecular Graphics and Modelling 2006, 25, 495-506.

(56) Bradley-Shaw, J. L.; Camp, P. J.; Dowding, P. J.; Lewtas, K. Self-assembly and friction of glycerol monooleate and its hydrolysis products in bulk and confined non-aqueous solvents. Physical Chemistry Chemical Physics 2018, 20, 17648-17657.

(57) Tang, Z.; Li, S. A review of recent developments of friction modifiers for liquid lubricants (2007-present). Current Opinion in Solid State and Materials Science 2014, 18, 119-139.

(58) Liu, P.; Lu, J.; Yu, H.; Ren, N.; Lockwood, F. E.; Wang, Q. J. Lubricant shear thinning behavior correlated with variation of radius of gyration via molecular dynamics simulations. The Journal of Chemical Physics 2017, 147, 84904.

(59) Cooks, D.; Townsend, H. Characterization of Iron Oxides Commonly formed as corrosion productes on steel. Hyperfine Interactions 2002, 103, 239-248.

(60) Humphrey, W.; Dalke, A.; Schulten, K. VMD: Visual Molecular Dynamics. Journal of Molecular Graphics 1996, 14, 33-38. 
(61) Siu, S. W.; Pluhackova, K.; Böckmann, R. A. Optimization of the OPLS-AA force field for long hydrocarbons. Journal of Chemical Theory and Computation 2012, 8, $1459-1470$.

(62) Jorgensen, W. L.; Maxwell, D. S.; Tirado-Rives, J. Development and testing of the OPLS all-atom force field on conformational energetics and properties of organic liquids. Journal of the American Chemical Society 1996, 118, 11225-11236.

(63) Ewen, J. P.; Gattinoni, C.; Thakkar, F. M.; Morgan, N.; Spikes, H. A.; Dini, D. A comparison of classical force-fields for molecular dynamics simulations of lubricants. Materials 2016, 9, 1-17.

(64) Berro, H.; Fillot, N.; Vergne, P. Molecular dynamics simulation of surface energy and ZDDP effects on friction in nano-scale lubricated contacts. Tribology International 2010, 43, 1811-1822.

(65) Blake, R. L.; Hessevick, R. E.; Zoltai, T.; Finger, L. W. Refinement of the hematite structure. American Mineralogist 1966, 51, 123-129.

(66) Ewen, J. P.; Gattinoni, C.; Morgan, N.; Spikes, H. A.; Dini, D. Nonequilibrium molecular dynamics simulations of organic friction modifiers adsorbed on iron oxide surfaces. Langmuir 2016, 32, 4450-4463.

(67) Latorre, C. A.; Ewen, J. P.; Gattinoni, C.; Dini, D. Simulating Surfactant-Iron Oxide Interfaces: From Density Functional Theory to Molecular Dynamics. Journal of Physical Chemistry B 2019, 123, 6870-6881.

(68) Plimpton, S. Fast Parallel Algorithms for Short-Range Molecular Dynamics. Journal of Computational Physics 1995, 117, 1-19.

(69) Verlet, L. Computer "Experiments" on Classical Fluids. I. Thermodynamical Properties of Lennard-Jones Molecules. Physical Review 1967, 159, 98. 
(70) Ryckaert, J. P.; Ciccotti, G.; Berendsen, H. J. C. Numerical-integration of Cartesian equations of motion of a system with constraints: molecular-dynamics of n-alkanes. Journal of Computational Physics 1977, 23, 327-341.

(71) Yeh, I. C.; Berkowitz, M. L. Ewald summation for systems with slab geometry. The Journal of Chemical Physics 1999, 111, 3155-3162.

(72) Fiorin, G.; Klein, M. L.; Henin, J. Using collective variables to drive molecular dynamics simulations. Molecular Physics 2013, 111, 3345-3362.

(73) Nosé, S. A Molecular-Dynamics Method for Simulations in the Canonical Ensemble. Mol. Phys. 1984, 52, 255-268.

(74) Hoover, W. G. Canonical Dynamics: Equilibrium Phase-Space Distributions. Phys. Rev. A 1985, 31, 1695-1697.

(75) Darve, E.; Pohorille, A. Calculating free energies using average force. Journal of Chemical Physics 2014, 9169, 9169-9183.

(76) Comer, J.; Gumbart, J. C.; Hénin, J.; Lelievre, T.; Pohorille, A.; Chipot, C. The adaptive biasing force method: Everything you always wanted to know but were afraid to ask. Journal of Physical Chemistry B 2015, 119, 1129-1151.

(77) Valleau, J. P.; Card, D. N. Monte Carlo Estimation of the Free Energy by Multistage Sampling. The Journal of Chemical Physics 1972, 57, 5457-5462.

(78) Park, S.; Khalili-Araghi, F.; Tajkhorshid, E.; Schulten, K. Free energy calculation from steered molecular dynamics simulations using Jarzynski's equality. The Journal of Chemical Physics 2003, 119, 3559-3566.

(79) Jaishankar, A.; Jusufi, A.; Vreeland, J. L.; Deighton, S. P.; Schilowitz, A. M. Correcting for solvent replacement effects in quartz crystal microbalance measurements. Sensors and Actuators, A: Physical 2018, 277, 60-64. 
(80) Zhang, Z.; Ahn, Y.; Son, J. Y.; Jang, J. Thermal Healing of the Nanometer-Wide Lines of Self-Assembled Monolayer. Journal of Physical Chemistry C 2016, 120, 1550915513.

(81) Pominov, A.; Müller-Hillebrand, J.; Träg, J.; Zahn, D. Interaction Models and Molecular Simulation Systems of Steel-Organic Friction Modifier Interfaces. Tribology Letters 2021, 69, 14.

(82) Smith, G. N.; Brown, P.; Rogers, S. E.; Eastoe, J. Evidence for a critical micelle concentration of surfactants in hydrocarbon solvents. Langmuir 2013, 29, 3252-3258.

(83) Nagarajan, R.; Ruckenstein, E. Theory of Surfactant Self-Assembly: A Predictive Molecular Thermodynamic Approach. Langmuir 1991, 7, 2934-2969.

(84) Fujii, Y.; Yamada, H.; Mizuta, M. Self-association of acetic acid in some organic solvents. The Journal of Physical Chemistry 1988, 92, 6768-6772.

(85) Bradley-Shaw, J. L.; Camp, P. J.; Dowding, P. J.; Lewtas, K. Glycerol monooleate reverse micelles in nonpolar solvents: Computer simulations and small-angle neutron scattering. Journal of Physical Chemistry B 2015, 119, 4321-4331.

(86) Shrestha, L. K.; Shrestha, R. G.; Varade, D.; Aramaki, K. Tunable Parameters for the Structural Control of Reverse Micelles in Glycerol Monoisostearate/Oil Systems: A SAXS Study. Langmuir 2009, 25, 4435-4442.

(87) Bradley-Shaw, J. L.; Camp, P. J.; Dowding, P. J.; Lewtas, K. Molecular Dynamics Simulations of Glycerol Monooleate Confined between Mica Surfaces. Langmuir 2016, 32, $7707-7718$.

(88) Danping, W.; Spikes, H. A. The lubricity of diesel fuels. Wear 1986, 111, 217-235.

(89) Wang, Y.; Hill, K.; Harris, J. G. Confined thin films of a linear and branched octane. A 
comparison of the structure and solvation forces using molecular dynamics simulations. The Journal of Chemical Physics 1994, 100, 3276-3285.

(90) Askwith, T. C.; Cameron, A.; Crouch, R. F. Chain Length of Additives in Relation to Lubricants in Thin Film and Boundary Lubrication. Proceedings of the Royal Society of London. Series A. Mathematical and Physical Sciences 1966, 291, 500-519.

(91) Machesky, M. L.; Ridley, M. K.; Biriukov, D. Oxalic Acid Adsorption on Rutile: Experiments and Surface Complexation Modeling to $150{ }^{\circ} \mathrm{C}$. Langmuir 2019, 35, $7631-7640$.

(92) Parambathu, A. V.; Wang, L.; Asthagiri, D.; Chapman, W. G. Apolar behavior of hydrated calcite (1014) surface assists in naphthenic acid adsorption. Energy and Fuels 2019, 33, 6119-6125.

(93) Sahoo, R. R.; Biswas, S. K. Frictional response of fatty acids on steel. Journal of Colloid and Interface Science 2009, 333, 707-718.

(94) Kuwahara, T.; Romero, P. A.; Makowski, S.; Weihnacht, V.; Moras, G.; Moseler, M. Mechano-chemical decomposition of organic friction modifiers with multiple reactive centres induces superlubricity of ta-C. Nature Communications 2019, 10, 1-11.

(95) Talbot, J.; Tarjus, G.; Van Tassel, P. R.; Viot, P. From car parking to protein adsorption: An overview of sequential adsorption processes. Colloids and Surfaces A: Physicochemical and Engineering Aspects 2000, 165, 287-324.

(96) Kawaguchi, M.; Yamamoto, M.; Nakamura, T.; Yamashita, M.; Kato, T.; Kato, T. Surface properties of mono-, di-, and triglycerol monostearate monolayers spread at the air-water interface. Langmuir 2001, 17, 4677-4680.

(97) Ries Jr., H. E.; Walker, D. C. Films of mixed horizontally and vertically oriented compounds. Journal of Colloid Science 1961, 374, 361-374. 
(98) Ramachandran, S.; Tsai, B. L.; Blanco, M.; Chen, H.; Tang, Y.; Goddard, W. A. Self-assembled monolayer mechanism for corrosion inhibition of iron by imidazolines. Langmuir 1996, 12, 6419-6428.

(99) Studt, P. Boundary lubrication: adsorption of oil additives on steel and ceramic surfaces and its influence on friction and wear. Tribology International 1989, 22, 111-119.

(100) Dietrich, H.; Zahn, D. Molecular Mechanisms of Solvent-Controlled Assembly of Phosphonate Monolayers on Oxide Surfaces. Journal of Physical Chemistry C 2017, 121, $18012-18020$.

(101) Krause, D. JUWELS: Modular Tier-0/1 Supercomputer at Jülich Supercomputing Centre. Journal of Large-Scale Research Facilities 2019, 5, A135. 


\section{Graphical TOC Entry}

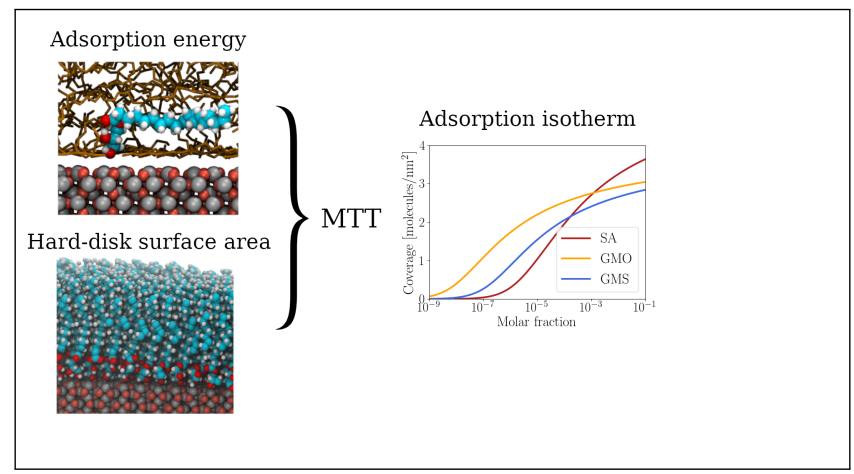

